\title{
SPECTRAL FAMILIES OF PROJECTIONS, SEMIGROUPS, AND DIFFERENTIAL OPERATORS
}

\author{
HAROLD BENZINGER, EARL BERKSON ${ }^{1}$ AND T. A. GILLESPIE
}

\begin{abstract}
This paper presents new developments in abstract spectral theory suitable for treating classical differential and translation operators. The methods are specifically geared to conditional convergence such as arises in Fourier expansions and in Fourier inversion in general. The underlying notions are spectral family of projections and well-bounded operator, due to D. R. Smart and J. R. Ringrose. The theory of well-bounded operators is considerably expanded by the introduction of a class of operators with a suitable polar decomposition. These operators, called polar operators, have a canonical polar decomposition, are free from restrictions on their spectra (in contrast to well-bounded operators), and lend themselves to semigroup considerations. In particular, a generalization to arbitrary Banach spaces of Stone's theorem for unitary groups is obtained. The functional calculus for well-bounded operators with spectra in a nonclosed arc is used to study closed, densely defined operators with a well-bounded resolvent. Such an operator $L$ is represented as an integral with respect to the spectral family of its resolvent, and a sufficient condition is given for $(-L)$ to generate a strongly continuous semigroup. This approach is applied to a large class of ordinary differential operators. It is shown that this class contains significant subclasses of operators which have a polar resolvent or generate strongly continuous semigroups. Some of the latter consist of polar operators up to perturbation by a semigroup continuous in the uniform operator topology.
\end{abstract}

1. Introduction. This paper is concerned with presenting new developments in abstract spectral theory, and their applications to classical translation and differential operators. The key notions are spectral family of projections and well-bounded operator due to D. R. Smart [23] and J. R. Ringrose [21]. Well-bounded operators are associated with certain monotone projection-valued functions on the real line $\mathbf{R}$, which are not given by projection-valued measures, and are suitable for treating conditional convergence such as arises in Fourier expansions. For our purposes a slightly stronger notion, that of well-bounded operator of type (B), is needed. Matters of background from the theory of well-bounded operators are discussed in concise form in $\$ 2$.

In $\$ 3$ we extend the theory of well-bounded operators to include operators with a suitable polar decomposition (polar operators). Polar operators are free from implicit requirements on the spectrum (in contrast to well-bounded operators), have a canonical decomposition with useful properties, and are well suited for applications to one-parameter semigroups ( $\$ \S 3$ and 4 ). In particular, in Theorem (4.20), we

Received by the editors September 14, 1981.

1980 Mathematics Subject Classification. Primary 47B40, 47D05, 47E05; Secondary 34B25, 42A45, $42 \mathrm{C} 20,43 \mathrm{~A} 50$.

'The work of the first and second authors was supported by a National Science Foundation Grant. 
obtain a generalization to arbitrary Banach spaces of Stone's theorem for unitary groups. This affords an abstract formulation of Fourier inversion phenomena in $L^{p}(\mathbf{R})$ and $L^{p}(\mathbf{T})$ for $1<p<\infty$, where $\mathbf{T}$ is the unit circle in the complex plane $\mathbf{C}$, $\mathbf{T}=\{z \in \mathbf{C}:|z|=1\}$ (see (4.47) below).

In $\$ 5$ the functional calculus for well-bounded operators, with spectra in a nonclosed arc in $\mathbf{C}$, on reflexive Banach spaces, is applied to the spectral analysis of closed, densely defined operators $L$ with well-bounded resolvent operators $R(\lambda, L)$. Specifically, $L$ is represented as an integral with respect to the spectral family of $R(\lambda, L)$. Further, a sufficient condition is given for $-L$ to generate a strongly continuous semigroup.

These results are applied to the study of a large class of ordinary differential operators defined for a compact interval, which are shown to have well-bounded resolvents. A rich structure theory for these operators is developed, and a subclass which generates strongly continuous semigroups is identified. Among these operators are significant classes with resolvents which are polar operators, or which generate semigroups which are polar up to perturbation by a uniformly continuous semigroup.

Throughout what follows $X$ will be a complex Banach space with dual space $X^{*}$, and $\mathscr{B}(X)$ will be the algebra of all bounded linear operators mapping $X$ into itself. We use "ker" to denote the kernel of a linear transformation. Our terminology concerning semigroups is that of [10, Chapter VIII], concerning $L^{p}$ multipliers that of $[11$, pp. 6,7]. The symbol $\mathbf{Z}$ will denote the set of all integers.

2. Relevant aspects of well-bounded operators summarized. In order to place the developments in succeeding sections in their proper context, we collect here, in a form tailored to our applications, the needed facts about well-bounded operators. For a full account see [9, Part 5].

Let $J=[a, b]$ be a compact interval of $\mathbf{R}$. Let $\operatorname{BV}(J)$ be the Banach algebra of complex-valued functions of bounded variation on $J$ with norm \|\|$_{J}$ defined by $\|f\|_{J}=|f(b)|+\operatorname{var}(f, J)$, where $\operatorname{var}(f, J)$ is the total variation of $f$ on $J$. Let $\mathrm{AC}(J)$ be the Banach subalgebra of $\mathrm{BV}(J)$ consisting of the absolutely continuous functions on $J$.

Definition. Let $T \in \mathscr{B}(X)$. $T$ is said to be well-bounded if there are a compact interval $J$ and a constant $K$ such that

$$
\|p(T)\| \leqslant K\|p\|_{J},
$$

for every polynomial $p$ with complex coefficients. In this case we say that $T$ is implemented by $(K, J)$.

Obviously, if $T$ is implemented by $(K, J)$, then the algebra homomorphism $p \mapsto p(T)$ on the subalgebra of $\mathrm{AC}(J)$ consisting of the polynomials extends to a continuous homomorphism from $\mathrm{AC}(J)$ into $\Re(X)$, denoted $f \mapsto f(T)$. J. R. Ringrose showed [21, Theorems 2 and 6] that well-bounded operators can be characterized by a "spectral theorem"-type of representation which associates with each well-bounded operator $T$ a certain kind of projection-valued function $E(\cdot)$ defined on $\mathbf{R} . E(\cdot)$ is called a decomposition of the identity for $T$. The characteristic properties of a 
decomposition of the identity are technically involved. Moreover, the values of a decomposition of the identity are projections acting in the dual space $X^{*}$, and a well-bounded operator need not have a unique decomposition of the identity. These technical complications of the general theory of well-bounded operators are removed when one considers the special class of well-bounded operators of type (B) (to be discussed presently) which are at the heart of the subject matter of this paper. For this reason we shall content ourselves with a short account (in Proposition (2.1) below) of some standard facts from the general theory of well-bounded operators, our purpose being largely to single out features of operators of type (B) which are actually part of the general theory.

(2.1) Proposition. Let $T$ be a well-bounded operator on $X$ implemented by $(K, J)$. Let $\sigma(T)$ be the spectrum of $T$, and let $E(\cdot)$ be a decomposition of the identity for $T$. Then

(i) $\sigma(T) \subseteq J$.

(ii) If $\alpha($ resp., $\beta)$ denotes $\min \sigma(T)$ (resp., $\max \sigma(T))$, then $T$ is implemented by $(K,[\alpha, \beta])$.

(iii) An open interval 9 is contained in $p(T)$, the resolvent set of $T$, if and only if $E(\cdot)$ is constant on 9 .

(iv) If there is a function $F(\cdot)$ on $\mathbf{R}$ whose values are projections acting in $X$ such that $F^{*}(\cdot)$ is a decomposition of the identity for $T$, then $T$ has a unique decomposition of the identity, and $\{T\}^{\prime}$, the commutant of $T$, equals $\{F(\lambda): \lambda \in \mathbf{R}\}^{\prime}$.

Proof. Conclusion (i) is an obvious consequence of the homomorphism from $\mathrm{AC}(J)$ into $\mathscr{B}(X)$ (see also [9, Corollary 15.9]). The proof of [9, Theorem 15.19] shows that there is some decomposition of the identity $G(\cdot)$ for $T$ such that $\|G(\lambda)\| \leqslant K$ for all $\lambda \in \mathbf{R}$, and

$$
\langle f(T) x, \phi\rangle=f(b)\langle x, \phi\rangle-\int_{a}^{b}\langle x, G(\lambda) \phi\rangle f^{\prime}(\lambda) d \lambda,
$$

for $x \in X, \phi \in X^{*}, f \in \mathrm{AC}[a, b]$, where $J=[a, b]$. It follows from [9, Theorem 19.2] that $G(\lambda)=0$ for $\lambda<\alpha$ and $G(\lambda)$ is the identity operator on $X^{*}$ for $\lambda \geqslant \beta$. Conclusion (ii) follows immediately. To see (iii), notice that we can assume without loss of generality that 9 is bounded. The "if" assertion follows from [9, Theorem 15.8(v)]. Conversely, suppose $G \subseteq \rho(T)$. Then by [9, Theorem 19.2] the projections $E(\lambda)$ for $\lambda \in \mathscr{G}$ all have the same range, and, since they commute, must be identical. Conclusion (iv) is part of [9, Theorem 16.3].

REMARK. It follows from Proposition (2.1)(ii) that if $T$ is a well-bounded operator whose spectrum consists of the single point $\lambda_{0}$, then $T=\lambda_{0} I$, where $I$ is the identity operator of $X$. In particular, a quasinilpotent well-bounded operator is 0 .

We shall call a well-bounded operator $T$ on $X$ decomposable in $X$ provided there is a function $E(\cdot)$ on $\mathbf{R}$ whose values are projections acting in $X$ such that $E^{*}(\cdot)$ is a decomposition of the identity for $T$. In this case it follows from Proposition (2.1)(iv) that $E(\cdot)$ is unique and $\{T\}^{\prime}=\{E(\lambda): \lambda \in \mathbf{R}\}^{\prime}$.

Definition. A well-bounded operator $T$ on $X$ is said to be of type (B) provided $T$ is decomposable in $X$, and the unique projection-valued function $E(\cdot)$ such that 
$E^{*}(\cdot)$ is a decomposition of the identity for $T$ further satisfies: (i) $E(\cdot)$ is right continuous on $\mathbf{R}$ in the strong operator topology, and (ii) $E(\cdot)$ has a left-hand limit in the strong operator topology at each point of $\mathbf{R}$.

The notion of well-bounded operator of type (B) gains much of its substance from the fact that a well-bounded operator on a reflexive space is automatically of type (B) [9, Theorem 17.17] (note, however, that [21,§6] contains an example of a well-bounded operator on a weakly complete space which is not of type (B)). It is obvious that once one specializes to the treatment of well-bounded operators of type (B), the emphasis can be placed on the projections $E(\lambda), \lambda \in \mathbf{R}$, acting in $X$ rather than on their adjoints. We now indicate briefly a framework for doing so.

(2.2) Definition. A spectral family in $X$ is a projection-valued function $F$ : $\mathbf{R} \rightarrow \mathscr{B}(X)$ which satisfies the following conditions.

(i) $\sup \{\|F(\lambda)\|: \lambda \in \mathbf{R}\}<+\infty$;

(ii) $F(\lambda) F(\mu)=F(\mu) F(\lambda)=F(\min \{\lambda, \mu\})$, for $\lambda, \mu \in \mathbf{R}$;

(iii) $F(\cdot)$ is right continuous on $\mathbf{R}$ in the strong operator topology;

(iv) $F(\cdot)$ has a left-hand limit in the strong operator topology at each point of $\mathbf{R}$;

(v) $F(\lambda) \rightarrow 0$ (resp., $F(\lambda) \rightarrow I$ ) in the strong operator topology as $\lambda \rightarrow-\infty$ (resp., $\lambda \rightarrow+\infty)$.

If there exist $a, b \in \mathbf{R}$ such that $F(\lambda)=0$ for $\lambda<a$ and $F(\lambda)=I$ for $\lambda \geqslant b$, we shall say that $F(\cdot)$ is concentrated on $[a, b]$.

REMARK. If $X$ is reflexive, then a projection-valued function $F: \mathbf{R} \rightarrow \mathscr{B}(X)$ satisfying (i) and (ii) of (2.2) automatically has a strong left-hand limit and a strong right-hand limit at each point of $\mathbf{R}$ (by, for instance, [1, Theorem 1]).

An integration theory is available for spectral families (for more details see [9, Chapter 17]). If $F(\cdot)$ is a spectral family in $X, J=[a, b]$ is a compact interval of $\mathbf{R}$, and $f \in \operatorname{BV}(J)$, then $\int_{J}^{r} f d F$ exists as the strong limit of Riemann-Stieltjes sums wherein the intermediate point in each subinterval is always taken to be the right end-point of the subinterval. If, in addition, $f$ is continuous on $J$, then $\int_{J} f d F$ exists as the strong limit of Riemann-Stieltjes sums. For $g \in \mathrm{BV}(J)$ we define $\int_{J}^{\oplus} g d F$ to be $g(a) F(a)+\int_{J}^{r} g d F$.

(2.3) Proposition. If $F(\cdot)$ is a spectral family in $X$, and $J$ is a compact interval of $\mathbf{R}$, define $\psi_{F, J}: \mathrm{BV}(J) \rightarrow \Re(X)$ by setting

$$
\psi_{F, J}(f)=\int_{J}^{\oplus} f d F, \quad \text { for } f \in \mathrm{BV}(J) .
$$

Then $\psi_{F, J}$ is an algebra homomorphism of $\mathrm{BV}(J)$ into $\Re(X)$, and

$$
\left\|\psi_{F, J}(f)\right\| \leqslant\|f\|_{J} \sup \{\|F(\lambda)\|: \lambda \in J\}, \quad f \in \operatorname{BV}(J) .
$$

REMARK. When there is no danger of confusion, we shall suppress the subscripts $F, J$ in the symbol $\psi_{F, J}$.

The next proposition can be seen from [9, proof of Theorem 17.14(iv)].

(2.4) Proposition. Let $T \in \mathscr{B}(X)$. Then $T$ is a well-bounded operator of type (B) if and only if there is a spectral family $E(\cdot)$ in $X$ such that

(i) $E$ is concentrated on a compact interval $[a, b]$ and

(ii) $T=\int_{[a, b]}^{\oplus} \lambda d E(\lambda)$. 
If this is the case, then for any spectral family $F(\cdot)$ satisfying (i) and (ii), $F^{*}(\cdot)$ is a decomposition of the identity for $T$ (in particular, the spectral family $E(\cdot)$ in (i) and (ii) above is uniquely determined).

Definition. If $T$ is a well-bounded operator of type (B), we call the unique spectral family in $X$ satisfying (i) and (ii) of Proposition (2.4) the spectral family of $T$.

REMARK. If $T$ is a well-bounded operator of type (B), and $E(\cdot)$ is its spectral family, then it is easy to see that for any compact interval $[A, B]$ containing $\sigma(T)$, $E(\cdot)$ must be concentrated on $[A, B]$ and $T=\int_{[A, B]}^{\oplus} \lambda d E(\lambda)$. Since we shall be concerned later on with eigenvalues of well-bounded operators of type (B), we list the next proposition for ready reference [9, Theorem 17.15(iii)].

(2.5) Proposition. Let $T$ be a well-bounded operator of type (B) on $X$, and let $E(\cdot)$ be its spectral family. Then for each $s \in \mathbf{R}, E(s)-E\left(s^{-}\right)$is a projection acting in $X$, and $\left\{E(s)-E\left(s^{-}\right)\right\} X=\{x \in X: T x=s x\}$.

In order to extend the theory of well-bounded operators so as to admit some operators with complex spectra, J. R. Ringrose, in [21, §8], introduced the notion of well-boundedness on a curve. Let $C$ be a simple, but not closed, rectifiable arc in the complex plane $\mathbf{C}$ (we shall call such an arc admissible). Let $C$ have the parametric representation $z=\gamma(s), 0 \leqslant s \leqslant \Lambda$, where $s$ is arc parameter on $C$.

(2.6) Definition [21]. Suppose $T \in \Re(X)$. We say that $T$ is well-bounded on $C$ provided there is a constant $K$ such that for every complex polynomial $p$

$$
\|p(T)\| \leqslant K\|p\|_{C}
$$

where $\|p\|_{C}=|p(\gamma(\Lambda))|+\operatorname{var}(p, C)$.

REMARKS. (i) The requirement imposed above that $C$ not be a closed curve is crucial to the usefulness of the notion defined in (2.6) (see [21, 8.1]).

(ii) Because the polynomials are dense in $Q_{C}$, the algebra of all absolutely continuous functions on $C$ equipped with \|\|$_{C}$ [21, Lemma 10], it is clear that if (2.7) holds for all polynomials $p$, then the algebra homomorphism $p \mapsto p(T)$ extends to an algebra homomorphism $f \mapsto f(T)$ of $\Theta_{C}$, and $\|f(T)\| \leqslant K\|f\|_{C}$. In particular, it follows that $\sigma(T)$ must be a subset of $C$.

The next proposition is contained in [21, 8.5].

(2.8) Proposition. Let $C, \gamma$ be as above, let $T \in \mathscr{B}(X)$, and suppose $T$ is well-bounded on $C$, with (2.7) holding for all polynomials $p$. Let $\Gamma$ be the inverse of $\gamma$, and set $A=\Gamma(T)$. Then $A$ is well-bounded (in fact, $A$ is implemented by $(K,[0, \Lambda])$ ). Moreover, $T=\gamma(A)$.

REMARK. Under the hypotheses of Proposition (2.8) $\sigma(T)=\gamma(\sigma(A))$, and $\sigma_{p}(T)$ $=\gamma\left(\sigma_{p}(A)\right)$, where " $\sigma_{p}$ " denotes point spectrum. To see this observe that there are sequences of polynomials $\left\{p_{n}\right\},\left\{q_{n}\right\}$ such that $\left\|p_{n}-\gamma\right\|_{[0, \Lambda]} \rightarrow 0$ and $\left\|q_{n}-\Gamma\right\|_{C}$ $\rightarrow 0$. So $\left\|p_{n}(A)-T\right\| \rightarrow 0$ and $\left\|q_{n}(T)-A\right\| \rightarrow 0$. The desired conclusions are now easily reached, using the Gelfand representation of a maximal abelian subalgebra of $\mathscr{B}(X)$ containing $T$ and $A$ to get the first conclusion. 
3. Polar operators. While Definition (2.6) generalizes the notion of well-bounded operator so that real spectrum is no longer a necessary condition, it still implicitly requires the spectrum to be contained in a suitable curve (thus not all normal operators on Hilbert space are well-bounded). In order to obtain a theory free from such limitations on the spectrum, we introduce the notion of polar operator.

(3.1) Definition. Let $T \in \mathscr{B}(X)$. We call $T$ a polar operator if there are operators $R$ and $A$ on $X$ such that $R$ and $A$ are commuting well-bounded operators of type (B), and $T=R e^{i A}$.

REMARKS. (i) It follows by [9, Theorem 16.17] that every scalar-type spectral operator (in particular, every normal operator) is polar.

(ii) The choice of a polar-type decomposition to define a more general notion than that of type (B) operator is natural from the standpoint of semigroup considerations, as will be apparent in $\S 4$ below. Additionally, it is known (see [12]) that a translation acting in $L^{p}(G)$ ( $G$ a locally compact abelian group, $\left.1<p<\infty\right)$ is of the form $e^{i A}$, where $A$ is well-bounded (consequently, since the underlying space is reflexive, $A$ is of type (B)). Thus even such a fundamental multiplier operator is covered in a natural way by the polar-type definition we employ (this theme is further elucidated in the examples at the end of $\S 4$ ). Note also that the translation operator corresponding to an element of infinite order in $G$ has spectrum equal to the unit circle [9. Theorem 20.18]; thus such an operator cannot have its spectrum contained in a simple nonclosed curve, and consequently cannot satisfy Definition (2.6).

The rest of this section will be devoted to the development of the theory of polar operators. In particular, it will be seen that such operators have a canonical decomposition (Theorem (3.16) below) and that for operators with real spectrum the notions of polar operator and of type (B) well-bounded operator are coextensive (Theorem (3.23)(ii) below). Throughout the remainder of this section, whenever $T, R$, and $A$ are as in Definition (3.1), we shall denote by $F(\cdot)$ and $E(\cdot)$ the spectral families of $R$ and $A$, respectively.

(3.2) ThEOREM. A polar operator is a generalized scalar operator (in the sense of [8]).

Proof. Let $T=R e^{i A}$, where $R$ and $A$ are as in Definition (3.1). By [8, Theorem 3.3, p. 106] it suffices to show that $R$ and $e^{i A}$ are both generalized scalar operators with commuting spectral distributions. Let $J_{1}$ (resp., $J_{2}$ ) be an interval of $\mathbf{R}$ such that $F(\cdot)($ resp., $E(\cdot))$ is concentrated on $J_{1}$ (resp., $J_{2}$ ), and

$$
R=\int_{J_{1}}^{\oplus} \lambda d F(\lambda) \quad\left(\text { resp., } A=\int_{J_{2}}^{\oplus} \lambda d E(\lambda)\right) .
$$

We have that $U_{f}=\left(f \mid J_{1}\right)(R)$, for $f \in C^{\infty}\left(\mathbf{R}^{2}\right)$, defines a spectral distribution for $R$, since $f \mid J_{1} \in \operatorname{AC}\left(J_{1}\right)$ and

$$
\left\|\left(f \mid J_{1}\right)(R)\right\|=O\left(\sup _{J_{1}}|f|+\sup _{J_{1}}\left|\frac{\partial f}{\partial x}\right|\right) .
$$

For real $s, e^{i s A}=\int_{J_{2}}^{\oplus} e^{i s \lambda} d E(\lambda)$, and so $\left\|e^{i s A}\right\|=O\left(1+\operatorname{var}\left(e^{i s(\cdot)}, J_{2}\right)\right)$. Thus $\left\|e^{i s A}\right\|=O(|s|)$ as $|s| \rightarrow \infty$. It follows from [8, Proposition 1.4 and Remark 1.5, pp. 
$130,131]$ that the equation

$$
V_{f}=\sum_{n=-\infty}^{\infty} \hat{f}(n) e^{i n A}, \quad \text { for } f \in C^{\infty}\left(\mathbf{R}^{2}\right),
$$

defines a spectral distribution for $e^{i A}$ (where we use $\hat{f}(n)$ to denote the $n$th Fourier coefficient of the restriction of $f$ to the unit circle). Since $R$ and $A$ commute it is clear that $U_{f} V_{g}=V_{g} U_{f}$ for $f, g \in C^{\infty}\left(\mathbf{R}^{2}\right)$, which gives the required result.

(3.3) Proposition. If $T$ is a polar operator, then there exist commuting type (B) well-bounded operators $R_{1}$ and $A_{1}$ such that $\sigma\left(R_{1}\right) \geqslant 0, T=R_{1} e^{i A_{1}}$, and $F_{1}(0)=$ $e^{i A_{1}} F_{1}(0)$, where $F_{1}(\cdot)$ is the spectral family of $R_{1}$.

Proof. Let $T=R e^{i A}$ as in Definition (3.1), and set $W=\{I-F(0)\} X, Y=$ $\left\{F(0)-F\left(0^{-}\right)\right\} X, Z=F\left(0^{-}\right) X$. Relative to $X=W \oplus Y \oplus Z$, define

$$
R_{1}=R|W \oplus 0| Y \oplus(-R \mid Z), \quad A_{1}=A|W \oplus 0| Y \oplus[(A+\pi I) \mid Z] .
$$

(Note that $W, Y, Z$ are $A$-invariant, since $A$ and $R$ commute.) Since $R \mid Y=0$ (by virtue of Proposition (2.5)), $R_{1} e^{i A_{1}}=R e^{i A}=T$, and $R_{1} A_{1}=A_{1} R_{1}$. Also, $\sigma\left(R_{1}\right) \geqslant 0$. To show that $R_{1}$ and $A_{1}$ are well-bounded of type (B), it clearly suffices to do this for the four operators $R|W, A| W,(-R) \mid Z$, and $(A+\pi I) \mid Z$. All but the third are clearly type (B) well-bounded operators. For $(-R) \mid Z$, define $G(\cdot)$ on $\mathbf{R}$ by setting

$$
G(\lambda)= \begin{cases}\left\{I-F\left((-\lambda)^{-}\right)\right\} \mid Z, & \text { for } \lambda \geqslant 0, \\ 0 \mid Z, & \text { for } \lambda<0 .\end{cases}
$$

Obviously, $G(\cdot)$ is a spectral family acting in $Z$. Pick $M>0$ so that $\sigma(R) \subseteq(-M, M]$. Clearly $G(\cdot)$ is concentrated on $[0, M]$. Set $S=\int_{[0, M]}^{\oplus} \lambda d G(\lambda)$. Thus $S$ is wellbounded of type (B) on $Z$, and $\sigma(S) \geqslant 0$. For $x \in Z, \phi \in X^{*}$, we have,

$$
\begin{aligned}
\langle S x, \phi\rangle & =M\langle x, \phi\rangle-\int_{0}^{M}\langle G(\lambda) x, \phi\rangle d \lambda \\
& =\int_{0}^{M}\left\langle F\left((-\lambda)^{-}\right) x, \phi\right\rangle d \lambda .
\end{aligned}
$$

By [9, Lemma 16.12], $F(\cdot) x$ has only a countable number of discontinuities on $\mathbf{R}$. It follows that

$$
\langle S x, \phi\rangle=\int_{0}^{M}\langle F(-\lambda) x, \phi\rangle d \lambda=\int_{-M}^{0}\langle F(\lambda) x, \phi\rangle d \lambda
$$

But

$$
\begin{aligned}
\langle R x, \phi\rangle & =M\langle x, \phi\rangle-\int_{-M}^{M}\langle F(\lambda) x, \phi\rangle d \lambda \\
& =M\langle x, \phi\rangle-\int_{0}^{M}\langle F(\lambda) x, \phi\rangle d \lambda-\langle S x, \phi\rangle .
\end{aligned}
$$

Since $x \in F\left(0^{-}\right) X, \int_{0}^{M}\langle F(\lambda) x, \phi\rangle d \lambda=M\langle x, \phi\rangle$. Thus $(-R) \mid Z=S$. Finally, it is easy to see that $Y=\operatorname{ker} R=\operatorname{ker} R_{1}=F_{1}(0) X$. It is immediate that $e^{i A_{1}} F_{1}(0)=F_{1}(0)$, and the proof of Proposition (3.3) is complete. 
A polar operator $T$ is, by Theorem (3.2), decomposable in the sense of [8], and so we can consider the spectral maximal subspaces $M_{T}(\delta)=\left\{x \in X: \sigma_{T}(x) \subseteq \delta\right\}$, for closed subsets $\delta$ of $\mathbf{C}$. For $0 \leqslant r \leqslant s$, we write $D_{r, s}$ for $\{z \in \mathbf{C}: r \leqslant|z| \leqslant s\}$.

(3.4) Proposition. Suppose $T=R e^{i A}$, as in Definition (3.1), and $\sigma(R) \geqslant 0$. Then for $0 \leqslant r \leqslant s$,

$$
M_{T}\left(D_{r, s}\right)=\left\{F(s)-F\left(r^{-}\right)\right\} X .
$$

Proof. Let $M=M_{T}\left(D_{r, s}\right)$. Then $M$ is a hyperinvariant subspace for $T$. In particular, $M$ is invariant under $R$ and $A$, and $\sigma(R \mid M) \subseteq \sigma(R)$ (this inclusion is also obvious by virtue of the fact that $\sigma(R)$ does not separate $\mathrm{C})$. Let $Q$ be a maximal abelian subalgebra of $\mathscr{B}(M)$ containing $R \mid M$ and $A \mid M$. Let $\lambda \in \sigma(R \mid M)$, and let $\phi$ be a homomorphism of $\mathbb{Q}$ onto $\mathrm{C}$, such that $\phi(R \mid M)=\lambda$. Then

$$
\phi(T \mid M)=\lambda \exp \{i \phi(A \mid M)\} \in \sigma(T \mid M) \subseteq D_{r, s},
$$

and so $\lambda \in[r, s]$. Thus $\sigma(R \mid M) \subseteq[r, s]$. Clearly, we now have

$$
\sigma_{R}(x) \subseteq \sigma(R \mid M) \subseteq[r, s] \text { for } x \in M
$$

Using [9, Theorem 19.3], we have from (3.5) that

$$
M \subseteq F(s) X \text {. }
$$

It is readily verified (compare the proof of Proposition (3.3)) that $(-R)$ is wellbounded of type (B) with spectral family $\lambda \mapsto I-F\left((-\lambda)^{-}\right)$, from which it follows easily by applying [9, Theorem 19.3] to (-R) that $\left\{I-F\left(r^{-}\right)\right\} X=\left\{x \in X: \sigma_{R}(x) \subseteq\right.$ $[r,+\infty)\}$. This equality, in conjunction with (3.5), gives $M \subseteq\left\{I-F\left(r^{-}\right)\right\} X$, and the inclusion just obtained together with (3.6) shows that

$$
M \subseteq\left\{F(s)-F\left(r^{-}\right)\right\} X .
$$

To obtain the reverse inclusion to (3.7), note first that $N=\left\{F(s)-F\left(r^{-}\right)\right\} X$ is $A$-invariant and $T$-invariant. Also $\sigma(R \mid N) \subseteq[r, s]$. By considering the Gelfand representation of a maximal abelian subalgebra of $\mathscr{B}(N)$ containing $R \mid N$ and $A \mid N$, it easily follows that $\sigma(T \mid N) \subseteq D_{r, s}$. The definition of $M$ now gives $N \subseteq M$ as required.

(3.8) Proposition. Suppose $T=R e^{i A}$ as in Definition (3.1), and $\sigma(R) \geqslant 0$. Then the following inclusion among commutants holds.

$$
\{T\}^{\prime} \subseteq\{F(\lambda): \lambda \in \mathbf{R}\}^{\prime} .
$$

Proof. For $s \geqslant 0, M_{T}\left(D_{0, s}\right)$ is hyperinvariant for $T$. Thus, by Proposition (3.4), $F(s) X$ is hyperinvariant for $T$. Similarly, by taking $s$ sufficiently large in Proposition (3.4), we get that for $r \geqslant 0,\left\{I-F\left(r^{-}\right)\right\} X$ is hyperinvariant for $T$. Since $F\left(\left(r+\frac{1}{n}\right)^{-}\right)$ $\rightarrow F(r)$ strongly as $n \rightarrow \infty$, we see that $\{I-F(r)\} X$ is hyperinvariant for $T$. If $S \in\{T\}^{\prime}$, it is now clear that $S F(s)=F(s) S$ for $s \geqslant 0$. This is trivially valid for $s<0$.

(3.9) Proposition. Suppose $T=R e^{i A}$ as in Definition (3.1), $\sigma(R) \geqslant 0$, and $F(0)=$ $e^{i A} F(0)$. Then $\{T\}^{\prime} \subseteq\left\{e^{i A}\right\}^{\prime}$. 
Proof. Let $S \in\{T\}^{\prime}$. By Proposition (3.8), $S \in\{F(\lambda): \lambda \in \mathbf{R}\}^{\prime}$. Hence

$$
R\left(e^{i A} S-S e^{i A}\right)=T S-S T=0 .
$$

Since $F(0) X=\operatorname{ker} R$, and since $F(0) S=S F(0)$, we have

$$
e^{i A} S-S e^{i A}=F(0)\left(e^{i A} S-S e^{i A}\right)=F(0) S-S F(0) e^{i A}=0,
$$

the last equality by virtue of the last hypothesis.

(3.10) Proposition. Let $T=R_{1} e^{i A_{1}}=R_{2} e^{i A_{2}}$, where, for $j=1,2$, the decomposition $T=R_{j} e^{i A_{j}}$ is as in Definition (3.1). Suppose further that $\sigma\left(R_{j}\right) \geqslant 0$ for $j=1,2$. Then

(i) $R_{1}=R_{2}$;

(ii) if $F_{j}(0) e^{i A_{j}}=F_{j}(0)$ for $j=1,2\left(\right.$ where $F_{j}(\cdot)$ is the spectral family of $\left.R_{j}\right)$, then $e^{i A_{1}}=e^{i A_{2}}$.

Proof. (i) By Proposition (3.8), $F_{1}(r) F_{2}(s)=F_{2}(s) F_{1}(r)$ for $r, s \in \mathbf{R}$. Moreover, Proposition (3.4) shows that $\left\{F_{1}(s)-F_{1}\left(r^{-}\right)\right\} X=\left\{F_{2}(s)-F_{2}\left(r^{-}\right)\right\} X$ for $0 \leqslant r \leqslant s$. It follows by commutativity that $F_{1}(s)-F_{1}\left(r^{-}\right)=F_{2}(s)-F_{2}\left(r^{-}\right), 0 \leqslant r \leqslant s$. Taking $r=0$ gives $F_{1}(\cdot)=F_{2}(\cdot)$, which proves (i).

(ii) Suppose $F_{j}(0) e^{i A_{j}}=F_{j}(0), j=1,2$. By (i) we can put $R=R_{1}=R_{2}$, and $F(\cdot)=F_{j}(\cdot), j=1,2$. We have $R\left(e^{i A_{1}}-e^{i A_{2}}\right)=0$. Hence

$$
e^{i A_{1}}-e^{i A_{2}}=F(0)\left(e^{i A_{1}}-e^{i A_{2}}\right)=0,
$$

as required.

(3.11) Proposition. If $T$ is a polar operator, there exist commuting type (B) well-bounded operators $R_{1}, A_{1}$ with $\sigma\left(R_{1}\right) \geqslant 0, \sigma\left(A_{1}\right) \subseteq[0,2 \pi], 2 \pi \notin \sigma_{p}\left(A_{1}\right)$ (where " $\sigma_{p}$ " denotes point spectrum $)$, and $F_{1}(0)=e^{i A_{1}} F_{1}(0)$ such that $T=R_{1} e^{i A_{1}}$, where $F_{1}(\cdot)$ is the spectral family of $R_{1}$.

Proof. By Proposition (3.3) there exist commuting type (B) well-bounded operators $R_{1}$ and $A$ such that $\sigma\left(R_{1}\right) \geqslant 0, T=R_{1} e^{i A}$, and $F_{1}(0)=e^{i A} F_{1}(0)$, where $F_{1}(\cdot)$ is the spectral family of $R_{1}$. We shall obtain a type (B) operator $A_{1}$ commuting with $R_{1}$ such that $\sigma\left(A_{1}\right) \subseteq[0,2 \pi], 2 \pi \notin \sigma_{p}\left(A_{1}\right)$ and $e^{i A_{1}}=e^{i A}$. To do this, let $k, m$ be integers with $k<0<m$ such that $E(2 \pi k)=0, E(2 \pi m)=I$, where $E(\cdot)$ is the spectral family of $A$. Set $X_{j}=\{E(2 \pi(j+1))-E(2 \pi j)\} X$, for $j=k, k+1, \ldots$, $(m-1)$, and let

$$
A_{0}=\bigoplus_{j=k}^{m-1}(A-2 \pi j I) \mid X_{j} .
$$

Obviously $A_{0}$ is a type (B) operator. Since each $X_{j}$ is $R_{1}$-invariant and $A R_{1}=R_{1} A$, we have that $A_{0}$ commutes with $R_{1}$. Moreover, $e^{i A_{0}}=e^{i A}$ and $\sigma\left(A_{0}\right) \subseteq[0,2 \pi]$. Let $E_{0}(\cdot)$ be the spectral family of $A_{0}$, and let $Y=\operatorname{ker}\left(A_{0}-2 \pi I\right)=\left\{I-E_{0}\left(2 \pi^{-}\right)\right\} X$. Put $A_{1}=\left[A_{0} \mid E_{0}\left(2 \pi^{-}\right) X\right] \oplus[0 \mid Y]$. It is clear that $A_{1}$ is a type (B) well-bounded operator, that $\sigma\left(A_{1}\right) \subseteq[0,2 \pi]$, and that $e^{i A_{1}}=e^{i A_{0}}=e^{i A}$. If $A_{1} x=2 \pi x$, then from the definition of $A_{1}$ we have

$$
A_{0} E_{0}\left(2 \pi^{-}\right) x=2 \pi x .
$$


From (3.12) we infer that $x \in E_{0}\left(2 \pi^{-}\right) X$, and so $A_{0} x=2 \pi x$, whence $x \in$ $\left\{I-E_{0}\left(2 \pi^{-}\right)\right\} X$. This shows that $2 \pi \notin \sigma_{p}\left(A_{1}\right)$. Since it is evident that $R_{1}$ commutes with $A_{1}$, the proof of the proposition is complete.

The construction of $A_{1}$ from $A_{0}$ in the proof of Proposition (3.11) gives the following proposition, which we record here for later use.

(3.13) Proposition. Suppose that $A_{0}$ is a type (B) well-bounded operator with spectral family $E_{0}(\cdot)$ such that $\sigma\left(A_{0}\right) \subseteq[0,2 \pi]$. Then there is a well-bounded operator $A_{1}$ of type (B) such that $\sigma\left(A_{1}\right) \subseteq[0,2 \pi], 2 \pi \notin \sigma_{p}\left(A_{1}\right), e^{i A_{1}}=e^{i A_{0}},\left\{A_{0}\right\}^{\prime} \subseteq\left\{A_{1}\right\}^{\prime}$, and

$$
\left\|E_{1}(\lambda)\right\| \leqslant 2 \sup \left\{\left\|E_{0}(s)\right\|: s \in \mathbf{R}\right\}+1, \text { for } \lambda \in \mathbf{R},
$$

where $E_{1}(\cdot)$ is the spectral family of $A_{1}$.

Given $0 \leqslant r \leqslant s \leqslant 2 \pi$, we shall write $L_{r, s}$ to denote $\left\{e^{i \theta}: r \leqslant \theta \leqslant s\right\}$. By Theorem (3.2), $e^{i A}$ is a generalized scalar operator, if $A$ is a well-bounded operator of type (B). We now identify the spectral maximal subspaces $M_{e^{i A}}\left(L_{r, s}\right), 0<r \leqslant s<2 \pi$, when $\sigma(A)$ satisfies the conclusion of Proposition (3.11). This allows us to obtain a desirable commutativity result.

(3.14) Proposition. Let $A$ be a type (B) well-bounded operator with spectral family $E(\cdot)$ such that $\sigma(A) \subseteq[0,2 \pi]$ and $2 \pi \notin \sigma_{p}(A)$. Then

(i) $M_{e^{i A}}\left(L_{r, s}\right)=\left\{E(s)-E\left(r^{-}\right)\right\} X$ for $0<r \leqslant s<2 \pi$;

(ii) $\left\{e^{i A}\right\}^{\prime}=\{A\}^{\prime}$.

Proof. (i) Fix $0<r \leqslant s<2 \pi$, and put $M=M_{e^{i A}}\left(L_{r, s}\right), N=\left\{E(s)-E\left(r^{-}\right)\right\} X$. $M$ is hyperinvariant for $e^{i A}$ and hence $A$-invariant. $N$ is clearly $A$-invariant. We have $\sigma(A \mid N) \subseteq[r, s]$. So by spectral mapping $\sigma\left(e^{i A} \mid N\right) \subseteq L_{r, s}$. Since $M$ is the spectral maximal subspace of $e^{i A}$ associated with $L_{r, s}, N \subseteq M$. If $M=\{0\}$ the desired conclusion is trivial. Otherwise we obtain $M \subseteq N$ as follows. Since $\sigma(A)$ does not separate the plane, $\sigma(A \mid M) \subseteq \sigma(A) \subseteq[0,2 \pi]$. Moreover, $\left\{e^{i \lambda}: \lambda \in \sigma(A \mid M)\right\}=$ $\sigma\left(e^{i A} \mid M\right)$, and the latter set is contained in $L_{r, s}$ (by [8, Theorem 15, p. 31]). It now follows that $\sigma(A \mid M) \subseteq[\dot{r}, s]$. Since obviously $A \mid M$ is a type (B) well-bounded operator with spectral family $E(\cdot) \mid M$, the desired inclusion follows easily, because $E(\cdot) \mid M$ must be concentrated on $[r, s]$.

(ii) Let $S \in \mathscr{B}(X)$ with $S e^{i A}=e^{i A} S$. By (i), $\left\{E(s)-E\left(r^{-}\right)\right\} X$ is invariant under $S$ for $0<r \leqslant s<2 \pi$. Since $2 \pi \notin \sigma_{p}(A), E\left(2 \pi^{-}\right)=I$. Let $s \rightarrow 2 \pi^{-}$and get $\left\{I-E\left(r^{-}\right)\right\} X$ is $S$-invariant for $0<r<2 \pi$. Let $\left\{r_{n}\right\}$ be a strictly decreasing sequence in $(r, 2 \pi)$ such that $r_{n} \rightarrow r$. Then $E\left(r_{n}^{-}\right) \rightarrow E(r)$ strongly, and so $\{I-E(r)\} X$ is $S$-invariant for $0<r<2 \pi$ (this holds trivially for $r=2 \pi$ ). In a similar fashion, if we fix $s$, and let $r \rightarrow 0^{+}$in $\left\{E(s)-E\left(r^{-}\right)\right\}$, it is seen that $\{E(s)-E(0)\} X$ is $S$-invariant for $0<s<2 \pi$.

Next we show that $E(0) X=\operatorname{ker}\left(e^{i A}-I\right)$. Since $E(0) X=\operatorname{ker} A$, it is obvious that $E(0) X \subseteq \operatorname{ker}\left(e^{i A}-I\right)$. Put $Y=\operatorname{ker}\left(e^{i A}-I\right)$. We can assume that $Y \neq\{0\}$. Obviously $Y$ is invariant under $E(\lambda)$ for all $\lambda \in \mathbf{R}$, and $A \mid Y$ is a type (B) well-bounded operator with $E(\cdot) \mid Y$ as its spectral family. 


$$
\left\{e^{i \lambda}: \lambda \in \sigma(A \mid Y)\right\}=\sigma\left(e^{i A} \mid Y\right)=\{1\} .
$$

Since $\sigma(A \mid Y) \subseteq[0,2 \pi]$, we have $\sigma(A \mid Y)$ is contained in the doubleton set $\{0,2 \pi\}$. In particular, by Proposition (2.1)(iii), $E(\cdot) \mid Y$ is constant on $(0,2 \pi)$. If this constant value were not $I \mid Y, 2 \pi$ would belong to $\sigma_{p}(A \mid Y)$. Thus $E(\cdot)|Y=I| Y$ on $[0,2 \pi]$. Obviously, then, $A|Y=0| Y$, and we have that $Y \subseteq \operatorname{ker} A$, which establishes our claim that $E(0) X=\operatorname{ker}\left(e^{i A}-I\right)$. Since $S$ commutes with $e^{i A}$, the latter set, and hence $E(0) X$, is $S$-invariant.

For $0<s<2 \pi$, the $S$-invariance of $\{E(s)-E(0)\} X$ and $E(0) X$ shows that $E(s) X$ is $S$-invariant. Since we have shown previously that $\{I-E(s)\} X$ is $S$ invariant, it now follows that $E(s)$ commutes with $S$ for $0<s<2 \pi$. Since $E(s) \rightarrow$ $E(0)$ strongly as $s \rightarrow 0^{+}$, it is clear that $S$ commutes with $E(\lambda)$ for all $\lambda \in \mathbf{R}$, and hence with $A$.

(3.15) Proposition. Let $A_{1}, A_{2}$ be well-bounded operators of type (B) such that for $j=1,2, \sigma\left(A_{j}\right) \subseteq[0,2 \pi]$ and $2 \pi \notin \sigma_{p}\left(A_{j}\right)$. Then $e^{i A_{1}}=e^{i A_{2}}$ implies $A_{1}=A_{2}$.

Proof. Suppose $e^{i A_{1}}=e^{i A_{2}}$, and for $j=1,2$, let $E_{j}(\cdot)$ be the spectral family of $A_{j}$. By Proposition (3.14)(ii), $E_{1}(r) E_{2}(s)=E_{2}(s) E_{1}(r)$ for all $r, s \in \mathbf{R}$. By Proposition (3.14)(i), $\left\{E_{1}(s)-E_{1}\left(r^{-}\right)\right\} X=\left\{E_{2}(s)-E_{2}\left(r^{-}\right)\right\} X$ for $0<r \leqslant s<2 \pi$. Thus $E_{1}(s)$ $-E_{1}\left(r^{-}\right)=E_{2}(s)-E_{2}\left(r^{-}\right)$for $0<r \leqslant s<2 \pi$, since the two sides of this equation represent commuting projections with the same range. Let $s \rightarrow 2 \pi^{-}$and get $E_{1}\left(r^{-}\right)=$ $E_{2}\left(r^{-}\right)$for $0<r<2 \pi$. It follows readily that $E_{1}(\cdot)=E_{2}(\cdot)$ on $(0,2 \pi)$, and hence on R. The proof of the proposition is complete.

We summarize the preceding results of this section in the next theorem.

(3.16) ThEOREM. Let $T$ be polar. Then Thas a decomposition

$$
T=R e^{i A}
$$

such that

(i) $R$ and $A$ are commuting well-bounded operators of type (B);

(ii) $\sigma(R) \geqslant 0$;

(iii) $F(0) e^{i A}=F(0)$, where $F(\cdot)$ is the spectral family of $R$;

(iv) $\sigma(A) \subseteq[0,2 \pi]$ and $2 \pi \notin \sigma_{p}(A)$.

Properties (i) and (ii) determine the operator $R$ in (3.17) uniquely; properties (i)-(iii) determine $e^{i A}$ uniquely; properties (i)-(iv) determine $A$ uniquely.

Definition. If $T$ is a polar operator, the unique decomposition (3.17) such that (i)-(iv) of Theorem (3.16) hold will be called the canonical decomposition of $T$.

(3.18) THEOREM. Let $T$ be a polar operator with canonical decomposition $T=R e^{i A}$. Then

(i) the commutants of $T, R$, and A satisfy $\{T\}^{\prime}=\{R\}^{\prime} \cap\{A\}^{\prime}$;

(ii) $T$ is quasinilpotent $\Leftrightarrow T=0 \Leftrightarrow R=0$;

(iii) let $\eta=\overline{(0,2 \pi) \cap \arg [\sigma(T) \backslash\{0\}]}$, where “-” denotes closure, and arg: $\mathbf{C} \backslash\{0\} \rightarrow \mathbf{R}$ is the branch of the argument function such that $0 \leqslant \arg z<2 \pi$. The 
chain of inclusions

$$
\eta \subseteq \sigma(A) \subseteq \eta \cup\{0\}
$$

holds.

Proof. Conclusion (i) is contained in Propositions (3.8), (3.9) and (3.14)(ii). The Gelfand theory, applied to a maximal abelian subalgebra of $\mathscr{B}(X)$ containing $R$ and $A$, shows that $\sigma(R)=\{|w|: w \in \sigma(T)\}$. Since a quasinilpotent well-bounded operator is 0 (see the remark following Proposition (2.1)), (ii) is evident.

We now turn our attention to the demonstration of (iii). If $T$ is quasinilpotent, then by (ii), (3.19) is trivial. So we assume that $\sigma(T) \backslash\{0\}$ is not empty. We first show

$$
\eta=\overline{(0,2 \pi) \cap \sigma(A)} .
$$

It is easy to see with the aid of Gelfand theory that $(0,2 \pi) \cap \arg [\sigma(T) \backslash\{0\}] \subseteq$ $\sigma(A) \cap(0,2 \pi)$. This gives $\eta \subseteq \overline{(0,2 \pi) \cap \sigma(A)}$. To obtain the reverse inclusion for (3.20), suppose $\lambda \in(0,2 \pi) \cap \sigma(A)$. Let $0<r<\lambda<s<2 \pi$. If $E(s)-E(r)=0$, then $E(\cdot)$ is constant on $(r, s)$ and $\lambda \in(r, s) \subseteq \rho(A)$. This contradicts the hypothesis on $\lambda$, and so $\{E(s)-E(r)\} X \neq 0$. Next observe that for some $\varepsilon>0$, $Y_{\varepsilon}=\{I-F(\varepsilon)\}\{E(s)-E(r)\} X \neq 0$. For, if no such $\varepsilon$ exists, then letting $\varepsilon \rightarrow 0^{+}$ we get $\{I-F(0)\}\{E(s)-E(r)\} X=0$. Consequently $\{E(s)-E(r)\} X \subseteq F(0) X$. Since $e^{i A} F(0)=F(0), F(0) X \subseteq \operatorname{ker}\left(e^{i A}-I\right)$. As shown in the proof of Proposition (3.14), $\operatorname{ker}\left(e^{i A}-I\right)=E(0) X$; so we have $\{E(s)-E(r)\} X \subseteq E(0) X$. This last inclusion implies $E(s)-E(r)=0$, which is false. Hence we shall choose $\varepsilon>0$ so that $Y_{\varepsilon}=\{I-F(\varepsilon)\}\{E(s)-E(r)\} X \neq 0$. Using [9, Theorem 16.2(ii)], we see that $\sigma\left(R \mid Y_{\varepsilon}\right) \subseteq \sigma(R \mid\{I-F(\varepsilon)\} X) \subseteq[\varepsilon,+\infty)$. Also we have

$$
\sigma\left(A \mid Y_{\varepsilon}\right) \subseteq \sigma(A \mid\{E(s)-E(r)\} X) \subseteq[r, s] .
$$

By Gelfand theory, the nonvoid set $\sigma\left(T \mid Y_{\varepsilon}\right)$ is contained in $\left\{\rho e^{i \theta}: \rho \geqslant \varepsilon\right.$ and $r \leqslant \theta \leqslant s\}$. Since $T \mid Y_{\varepsilon}$ is a direct summand of $T, \sigma\left(T \mid Y_{\varepsilon}\right) \subseteq \sigma(T)$. Thus $\arg [\sigma(T) \backslash\{0\}]$ intersects $[r, s]$. In view of the arbitrariness of $r$ and $s$, we have shown

$$
(0,2 \pi) \cap \sigma(A) \subseteq \eta .
$$

In particular, (3.20) is established. The first inclusion in (3.19) is immediate from (3.20). In view of (3.21) the second inclusion in (3.19) holds if we show the implication $2 \pi \in \sigma(A) \Rightarrow 2 \pi \in \eta$. Suppose $2 \pi \in \sigma(A)$. If some open interval $(r, 2 \pi)$, $0<r<2 \pi$, does not intersect $\sigma(A)$, then $E(\cdot)$ is constant on $(r, 2 \pi)$. This constant value of $E(\cdot)$ is $E\left(2 \pi^{-}\right)=I$. It follows that $2 \pi \in \rho(A)$, contradicting our hypothesis. Thus $2 \pi \in \overline{\sigma(A) \cap(0,2 \pi)}$. By (3.20) the proof of the theorem is complete.

For convenience we record in the next proposition a fact mentioned in the proof of Theorem (3.18)(ii).

(3.22) Proposition. Let $T$ be a polar operator with canonical decompositon $T=$ $R e^{i A}$. Then $\sigma(R)=\{|w|: w \in \sigma(T)\}$. 
(3.23) THEOREM. Let $T$ be a polar operator. Then

(i) $T$ is of the form $e^{i A}$, where $A$ is well-bounded of type (B), if and only if $\sigma(T) \subseteq \mathbf{T}$, where $\mathbf{T}$ is the unit circle $\{z \in \mathbf{C}:|z|=1\}$.

(ii) $T$ is well-bounded of type (B) if and only if $\sigma(T) \subseteq \mathbf{R}$.

Proof. (i) Suppose $\sigma(T) \subseteq \mathbf{T}$, and let $T=R e^{i A}$ be the canonical decomposition of $T$. By Proposition (3.22) $\sigma(R)=\{1\}$. Since $R$ is well bounded, it follows by Proposition (2.1)(ii) that $R=I$.

(ii) The "only if" assertion is evident. Conversely, suppose $\sigma(T) \subseteq \mathbf{R}$, and let $T=R e^{i A}$ be the canonical decomposition for $T$. Let $F(\cdot)$ and $E(\cdot)$ be the spectral families of $R$ and $A$, respectively. By Theorem (3.18)(iii) $\sigma(A)$ is contained in the doubleton set $\{0, \pi\}$. It is easy to see from this that $E(\cdot)=E(0)$ on $[0, \pi)$, and $E(\cdot)=I$ on $[\pi, 2 \pi]$. So $A=\pi(I-E(0))$. Let $P=I-E(0)=E(\pi)-E\left(\pi^{-}\right)$. Let $X_{1}=P X=\{x \in X: A x=\pi x\}$, and $X_{2}=(I-P) X=\operatorname{ker} A$. It is now easy to see that $T=\left[(-R) \mid X_{1}\right] \oplus R \mid X_{2}$. Since each of the direct summands is a well-bounded operator of type (B) on the indicated subspace, $T$ is well-bounded of type (B) on $X$, as required.

We conclude this section with a theorem which affords a link between well-bounded operators on curves and polar operators. We continue to use "arg" as described in Theorem (3.18)(iii).

(3.24) ThEOREM. Let $X$ be reflexive, let $C$ be an admissible arc, and let $T \in \mathscr{B}(X)$ be well-bounded on $C$. Let $C$ have the parametric representation $z=\gamma(s), 0 \leqslant s \leqslant \Lambda$, where $s=$ arc parameter. Suppose

(i) there is a partition $s_{0}=0<s_{1}<\cdots<s_{n}=\Lambda$ of $[0, \Lambda]$ such that on each closed subinterval $\left[s_{j-1}, s_{j}\right], j=1,2, \ldots, n,|\gamma(\cdot)|$ is monotone;

(ii) there is a partition $u_{0}=0<u_{1}<\cdots<u_{m}=\Lambda$ such that on each open subinterval $\left(u_{j-1}, u_{j}\right), j=1,2, \ldots, m, \arg \gamma(\cdot)$ is monotone.

Then $T$ is a polar operator. (Note: in each of (i) and (ii) above we do not assume the same trend (increasing or decreasing) from subinterval to subinterval.)

Proof. By Proposition (2.8) there is a well-bounded operator $W$ implemented by $(K,[0, \Lambda]$ ) (for some constant $K$ ) such that $T=\gamma(W)$. Since $X$ is reflexive $W$ is of type (B). Let $H(\cdot)$ be the spectral family of $W$, let $J=[0, \Lambda]$, and let $\psi\left(=\psi_{H, J}\right)$ be the homomorphism of $\operatorname{BV}(J)$ in Proposition (2.3). Then clearly $\psi(\gamma)=T$. If the origin lies on $C$, then $\gamma$ assumes the value 0 at only one point $\tilde{u}$, which must be one of the partition points $u_{j}$ in (ii). In this case, as a convenience, we set $\arg \gamma(\tilde{u})=0$. In any event, $\arg \gamma(\cdot) \in \operatorname{BV}(J)$ and $\gamma=|\gamma| e^{i \arg \gamma}$ on $J$. Thus $T=[\psi(|\gamma|)] e^{i \psi(\arg \gamma)}$. Let $R=\psi(|\gamma|), A=\psi(\arg \gamma)$. Obviously $R$ and $A$ commute. To complete the proof we show that $R$ and $A$ are well-bounded. To see this for $R$, let $p$ be any polynomial. Then

$$
\|p(R)\|=\|\psi(p(|\gamma|))\| \leqslant K\|p(|\gamma|)\|_{J} .
$$

Because of the montonicity assumption in (i), we have for $j=1,2, \ldots, n$ that $\operatorname{var}\left(p(|\gamma|),\left[s_{j-1}, s_{j}\right]\right) \leqslant \operatorname{var}(p,[0, M])$, where $M=\sup _{J}|\gamma|$. Thus $\|p(|\gamma|)\|_{J} \leqslant$ $\sup _{[0, M]}|p|+n \operatorname{var}(p,[0, M])$. In conjunction with (3.25) this shows that $R$ is 
well-bounded. Similarly,

$$
\|p(A)\| \leqslant K\|p(\arg \gamma)\|_{J} .
$$

On each subinterval $\left[u_{j-1}, u_{j}\right]$ in (ii) arg $\gamma$ has a right-hand limit $\alpha_{j}$ at $u_{j-1}$ and a left-hand limit $\tilde{\alpha}_{j}$ at $u_{j}$. It is easy to see that

$$
\begin{aligned}
\operatorname{var}\left(p(\arg \gamma),\left[u_{j-1}, u_{j}\right]\right) \leqslant & \operatorname{var}(p,[0,2 \pi])+\left|p\left(\alpha_{j}\right)-p\left(\arg \gamma\left(u_{j-1}\right)\right)\right| \\
& +\left|p\left(\tilde{\alpha}_{j}\right)-p\left(\arg \gamma\left(u_{j}\right)\right)\right| .
\end{aligned}
$$

Thus we have

$$
\operatorname{var}\left(p(\arg \gamma),\left[u_{j-1}, u_{j}\right]\right) \leqslant 3 \operatorname{var}(p,[0,2 \pi]) .
$$

It is immediate from (3.27) that

$$
\|p(\arg \gamma)\|_{J} \leqslant \sup _{[0,2 \pi]}|p|+3 m \operatorname{var}(p,[0,2 \pi]) .
$$

This, in conjunction with (3.26), shows that $A$ is well-bounded, as required.

4. One-parameter semigroups of polar operators. Suppose that $\left\{T_{t}\right\}, t \geqslant 0$, is a strongly continuous semigroup of polar operators, that each $T_{t}$ has the canonical decomposition $R_{t} e^{i A_{t}}$, and that the spectral families of the $A_{t}$ are uniformly bounded. In this section we show that the families $\left\{R_{t}\right\}$ and $\left\{e^{i A_{t}}\right\}, t \geqslant 0$, are necessarily strongly continuous semigroups. We then investigate the structure of the latter two semigroups.

(4.1) LEMMA. Suppose $S_{1}$ and $S_{2}$ are commuting well-bounded operators of type (B) on $X$, and $Q$ is a quasinilpotent operator on $X$ such that $S_{1}=S_{2}+Q$. Then $Q=0$.

Proof. Let $G_{k}(\cdot)$ be the spectral family of $S_{k}, k=1,2$. Let $i$ be one of the indices 1,2 , and $j$ the other index. If $\lambda \in \mathbf{R}$ and $x=G_{i}(\lambda) x$, then clearly

$$
\sigma_{S_{j}}(x) \subseteq \sigma\left(S_{j} \mid G_{i}(\lambda) X\right)=\sigma\left(S_{i} \mid G_{i}(\lambda) X\right) .
$$

By [9, Theorems 16.2(ii) and 19.3], we get from (4.2) that $G_{1}(\lambda) X=G_{2}(\lambda) X$ for $\lambda \in \mathbf{R}$. By commutativity $G_{1}(\cdot)=G_{2}(\cdot)$, and the lemma is shown.

(4.3) LEMMA. Let $G$ be a spectral family concentrated on the compact interval $[a, b]$, let $f$ be a strictly increasing continuous function on $[a, b]$, and let $g:[f(a), f(b)] \rightarrow[a, b]$ be the inverse of $f$. Then $\int_{[a, b]}^{\oplus} f(\lambda) d G(\lambda)$ is a well-bounded operator of type (B) with spectral family $H(\cdot)$ given by

$$
H(\lambda)= \begin{cases}I, & \text { for } \lambda \geqslant f(b), \\ G(g(\lambda)), & \text { for } \lambda \in[f(a), f(b)], \\ 0, & \text { for } \lambda<f(a) .\end{cases}
$$

Proof. It is easy to check directly that (4.4) defines a spectral family $H(\cdot)$ concentrated on $[f(a), f(b)]$, and that

$$
\int_{[a, b]}^{\oplus} f(\lambda) d G(\lambda)=\int_{[f(a), f(b)]}^{\oplus} \mu d H(\mu) .
$$


(4.5) THEOREM. Let $\left\{T_{t}\right\}, t \geqslant 0$, be a strongly continuous one-parameter semigroup of polar operators on $X$. For $t \geqslant 0$ let $T_{t}$ have the canonical decomposition $T_{t}=R_{t} e^{i A_{t}}$, and let $F_{t}(\cdot)$ (resp., $\left.E_{t}(\cdot)\right)$ be the spectral family of $R_{t}\left(\right.$ resp., $\left.A_{t}\right)$. Then if

$$
\sup \left\{\left\|E_{t}(\lambda)\right\|: t \geqslant 0, \lambda \in \mathbf{R}\right\}<+\infty
$$

we have

(i) the families $\left\{R_{t}\right\}_{t \geqslant 0}$ and $\left\{e^{i A_{t}}\right\}_{t \geqslant 0}$ are strongly continuous semigroups;

(ii) for $t \geqslant 0, R_{t}$ is one-to-one;

(iii) For $t>0 R_{t}=\int_{[0, \beta]}^{\oplus} \lambda^{t} d F_{1}(\lambda)$, where $\beta=\max \sigma\left(R_{1}\right)$.

Proof. The demonstration is modeled after a proof by A. Sourour for semigroups of scalar-type operators [24, proof of Theorem (4.4)]. However, in adapting Sourour's proof to the present situation, considerable care is necessary, because the tools for handling well-bounded operators differ markedly from those for scalar-type operators.

Notice that

$$
\begin{aligned}
\left\{R_{t}: t \geqslant 0\right\} & \cup\left\{A_{t}: t \geqslant 0\right\} \cup\left\{T_{t}: t \geqslant 0\right\} \\
& \cup\left\{E_{t}(\lambda): t \geqslant 0, \lambda \in \mathbf{R}\right\} \cup\left\{F_{t}(\lambda): t \geqslant 0, \lambda \in \mathbf{R}\right\}
\end{aligned}
$$

is a commutative family. Since $R_{0} e^{i A_{0}}$ is the canonical decomposition of $I$, we have trivially that $R_{0}=I, A_{0}=0$. For $t \geqslant 0$, we let $U_{t}=e^{i A_{t}}$. For $n$ a positive integer and $t \geqslant 0, R_{t} e^{i A_{t}}=R_{t / n}^{n} e^{i n A_{t / n}}$. By Lemma (4.3) and Proposition (3.10)(i),

$$
R_{t}=R_{t / n}^{n} \text { and } F_{t}(\lambda)=F_{t / n}\left(\lambda^{1 / n}\right) \text { for } \lambda \geqslant 0,
$$

whenever $t \geqslant 0$ and $n$ is a positive integer. If $x \in X, u>0$, and $T_{u} x=0$, then $F_{u}(0) x=x$. By $(4.6), F_{u / n}(0) x=x$, and so $T_{u / n} x=0$ for every positive integer $n$. By strong continuity of $\left\{T_{t}\right\}, x=0$. Thus $T_{t}$ is one-to-one for $t \geqslant 0$, which is equivalent to conclusion (ii) of the theorem. So $F_{t}(0)=0$ for $t \geqslant 0$. We write $\psi$ for the homomorphism of $\mathrm{BV}[0, \beta]$ corresponding to $F_{1}(\cdot)$ and $[0, \beta]$ as in Proposition (2.3). For $t>0$, let $f_{t}:[0, \beta] \rightarrow \mathbf{R}$ be given by $f_{t}(\lambda)=\lambda^{t}$. Set $S_{t}=\psi\left(f_{t}\right)$ for $t>0$ and $S_{0}=I$. Clearly $\left\{S_{t}\right\}, t \geqslant 0$, is a semigroup, and $\sigma\left(S_{t}\right) \geqslant 0$ for $t \geqslant 0$. Moreover, the penultimate conclusion of $\left[9\right.$, Theorem 17.16] shows that $\left\{S_{t}\right\}$ is strongly continuous on $[0,+\infty)$. It follows from Lemma (4.3) that $S_{t}$ is well-bounded of type (B) for $t>0$ with spectral family $H_{t}(\cdot)$ satisfying

$$
H_{t}(\lambda)=F_{1}\left(\lambda^{1 / t}\right) \text { for } \lambda \geqslant 0 .
$$

Use of (4.7), in conjunction with (4.6), shows that $F_{1 / n}(\lambda)=H_{1 / n}(\lambda)$ for $\lambda \geqslant 0, n$ a positive integer. Hence $S_{1 / n}=R_{1 / n}$ for any positive integer $n$. If $m$ is a positive integer, then $T_{m / n}=T_{1 / n}^{m}$, and application of a previous argument shows that $R_{m / n}=R_{1 / n}^{m}$. Thus

$$
R_{t}=S_{t}, \text { for } t \text { rational, } t \geqslant 0 \text {. }
$$

For each positive integer $n$ let $X_{n}=\left[I-F_{1}(1 / n)\right] X$, and put $X_{0}=\cup_{n=1}^{\infty} X_{n}$. Note that $\left\{X_{n}\right\}_{n=1}^{\infty}$ is an increasing sequence of subspaces, and $X_{0}$ is a dense linear manifold in $X$. Observe also that by virtue of (4.7) $S_{t}$ is one-to-one for $t \geqslant 0$. It is easy to see that for $t \geqslant 0$ and $n$ a positive integer $X_{n}$ is invariant under $S_{t}$, and $S_{t} \mid X_{n}$ 
is invertible in $\mathscr{B}\left(X_{n}\right)$. In particular $X_{0} \subseteq S_{t} X$ for $t \geqslant 0$. For $t \geqslant 0$ we define the linear transformation $V_{t}: X_{0} \rightarrow X$ by setting $V_{t}=\left(T_{t} S_{t}^{-1}\right) \mid X_{0}$. From (4.8) we have $V_{t}=U_{t} \mid X_{0}$ for $t$ rational, $t \geqslant 0$. Let $t_{0}$ be nonnegative and $\left\{t_{k}\right\}_{k=1}^{\infty}$ a sequence of nonnegative rationals convergent to $t_{0}$. Fix a positive integer $n$. For each $k$ define $h_{k} \in \mathrm{AC}[0, \beta]$ by setting $h_{k}(\lambda)=n^{t_{k}}$ for $0 \leqslant \lambda \leqslant n^{-1}$, and $h_{k}(\lambda)=\lambda^{-t_{k}}$ for $n^{-1} \leqslant \lambda$ $\leqslant \beta$. Similarly define $h_{0}$ by replacing $t_{k}$ by $t_{0}$ in the foregoing. It is easy to see that if $x \in X_{n}$, then $S_{t_{k}}^{-1} x=\psi\left(h_{k}\right) x$ for all $k$, and $S_{t_{0}}^{-1} x=\psi\left(h_{0}\right) x$. Again we use the penultimate conclusion in [9, Theorem 17.16] to get

$$
S_{t_{k}}^{-1} x \rightarrow S_{t_{0}}^{-1} x \text { for all } x \in X_{0} \text {, whenever } t_{0} \geqslant 0 \text { and }
$$

$\left\{t_{k}\right\}$ is a sequence of nonnegative rationals convergent to $t_{0}$.

With $\left\{t_{k}\right\}, t_{0}$ as in (4.9), it follows from the strong continuity of $\left\{T_{t}\right\}_{t \geqslant 0}$ that $\left\{\left\|T_{t_{k}}\right\|\right\}$ is uniformly bounded. Thus we have for $x \in X_{0}, V_{t_{k}} x=T_{t_{k}} S_{t_{k}}^{-1} x \rightarrow V_{t_{0}} x$. Since each $t_{k}$ is rational, $V_{t_{k}} x=U_{t_{k}} x$, and so

$$
U_{t_{k}} x \rightarrow V_{t_{0}} x \quad \text { for all } x \in X_{0} \text {, whenever }\left\{t_{k}\right\}, t_{0} \text { are as in (4.9). }
$$

For each $t \geqslant 0, U_{t}=e^{i A_{t}}=\int_{[0,2 \pi]}^{\oplus} e^{i \lambda} d E_{t}(\lambda)$. Thus we have

$$
\left\|U_{t}\right\| \leqslant K[1+2 \pi] \text { for } t \geqslant 0 \text {, where } K=\sup \left\{\left\|E_{t}(\lambda)\right\|: t \geqslant 0, \lambda \in \mathbf{R}\right\} .
$$

Since $X_{0}$ is dense in $X$, it follows from (4.10) and (4.11) that

for each $t_{0} \geqslant 0, V_{t_{0}}$ has a unique extension to a bounded linear operator $\tilde{V}_{t_{0}}$ on $X$; if $\left\{t_{k}\right\}$ is any sequence of nonnegative rationals convergent to $t_{0}$, then $U_{t_{k}} \rightarrow \tilde{V}_{t_{0}}$ strongly on $X$.

From the characterization of $\tilde{V}_{t_{0}}$ by strong convergence given in (4.12), it is clear that for $t \geqslant 0 \tilde{V}_{t}$ commutes with $S_{r}$ and $T_{r}$ for all $r \geqslant 0$, and with $\left\{F_{1}(\lambda): \lambda \in \mathbf{R}\right\}$. If $x \in X_{0}, r \geqslant 0$, and $t \geqslant 0$, then

$$
\tilde{V}_{t+r} x=T_{t+r} S_{t+r}^{-1} x=T_{t} T_{r}\left(S_{r}^{-1} S_{t}^{-1} x\right)=T_{t} V_{r} S_{t}^{-1} x
$$

Hence $\tilde{V}_{t+r} x=T_{t} S_{t}^{-1} V_{r} x=V_{t} V_{r} x$. So $\tilde{V}_{t+r}=\tilde{V}_{t} \tilde{V}_{r}$ for $r, t \geqslant 0$. Since $V_{t}=U_{t} \mid X_{0}$ for $t$ rational and nonnegative, $\tilde{V}_{0}=I$, and so $\left\{\tilde{V}_{t}\right\}_{t \geqslant 0}$ is a semigroup.

Next we show that $\sigma\left(\tilde{V}_{t_{0}}\right) \subseteq \mathbf{T}$ for $t_{0} \geqslant 0$. Let $\sigma_{a}\left(\tilde{V}_{t_{0}}\right)$ be the approximate point spectrum of $\tilde{V}_{t_{0}}$. For some $s \geqslant 0, t_{0}+s$ is rational; hence $\tilde{V}_{t_{0}} \tilde{V}_{s}=U_{t_{0}+s}$. Since $U_{t_{0}+s}$ is invertible, and $\tilde{V}_{t_{0}}$ commutes with $\tilde{V}_{s}, 0 \in \rho\left(\tilde{V}_{t_{0}}\right)$. Now let $\mu \in \mathbf{C} \backslash \mathbf{T}$. For all $t \geqslant 0$,

$$
\left(\mu-U_{t}\right)^{-1}=\int_{[0,2 \pi]}^{\oplus}\left(\mu-e^{i \lambda}\right)^{-1} d E_{t}(\lambda) .
$$

So we have $\left\|\left(\mu-U_{t}\right)^{-1}\right\| \leqslant K\left\|\left(\mu-e^{i \lambda}\right)^{-1}\right\|_{[0,2 \pi]}$. It follows that for $\mu \in \mathbf{C} \backslash \mathbf{T}$ there is a constant $M_{\mu}$ such that $\left\|\left(\mu-U_{t}\right)^{-1}\right\| \leqslant M_{\mu}$ for all $t \geqslant 0$. Let $\left\{t_{n}\right\}$ be a sequence of nonnegative rationals convergent to $t_{0}$. By (4.12) $U_{t_{n}} \rightarrow \tilde{V}_{t_{0}}$ strongly on $X$. For $x \in X$, $\|x\| \leqslant M_{\mu}\left\|\left(\mu-U_{t_{n}}\right) x\right\|$ for all $n$. Thus $\sigma_{a}\left(\tilde{V}_{t_{0}}\right)$ is disjoint from $\mathbf{C} \backslash \mathbf{T}$. Suppose there were a point $\zeta$ in $\sigma\left(\tilde{V}_{t_{0}}\right) \cap[\mathbf{C} \backslash \mathbf{T}]$. Let $\mathcal{G}$ be the component of $\mathbf{C} \backslash \mathbf{T}$ containing $\zeta$. Since $0 \in \rho\left(\tilde{V}_{t_{0}}\right), \mathcal{G}$ contains a point $w$ of $\rho\left(\tilde{V}_{t_{0}}\right)$. Join $\zeta$ to $w$ by a curve $C$ contained in $\mathcal{G}$. Obviously $C$ contains a point in the boundary of $\sigma\left(\tilde{V}_{t_{0}}\right)$. Since the boundary of $\sigma\left(\tilde{V}_{t_{0}}\right)$ is contained in $\sigma_{a}\left(\tilde{V}_{t_{0}}\right)$, we have reached a contradiction. So $\sigma\left(\tilde{V}_{t}\right) \subseteq \mathbf{T}$ for $t \geqslant 0$. 
For $t \geqslant 0$, we have, using the definition of $V_{t}$,

$$
R_{t} U_{t}=T_{t}=\tilde{V}_{t} S_{t}=S_{t} \tilde{V}_{t}
$$

Obviously $\left\{R_{t}, U_{t}, S_{t}, \tilde{V}_{t}\right\}$ is a commutative family, and it further follows from (4.13) that $R_{t}=S_{t}+Q_{t}$ where $Q_{t}$ is quasinilpotent. By Lemma (4.1), $R_{t}=S_{t}$ for $t \geqslant 0$. From what has already been shown for $\left\{S_{t}\right\}_{t \geqslant 0}$, we have that $\left\{R_{t}\right\}_{t \geqslant 0}$ is a strongly continuous semigroup, and conclusion (iii) of the theorem holds. It remains to show that $\left\{U_{t}\right\}_{t \geqslant 0}$ is a strongly continuous semigroup. From (4.13) we now see that $R_{t}\left(U_{t}-\tilde{V}_{t}\right)=0$ for $t \geqslant 0$. In view of conclusion (ii), we get $U_{t}=\tilde{V}_{t}$ for $t \geqslant 0$. In particular $\left\{U_{t}\right\}_{t \geqslant 0}$ is a semigroup. Next suppose that $t_{0} \geqslant 0$ and $\left\{t_{k}\right\}_{k=1}^{\infty}$ is a sequence of nonnegative numbers convergent to $t_{0}$. The argument used to establish (4.10) can now be employed to show that $V_{t_{k}} x \rightarrow V_{t_{0}} x$ for all $x \in X_{0}$. Hence $U_{t_{k}} x \rightarrow U_{t_{0}} x$ for $x \in X_{0}$. It now follows from (4.11) that $\left\{U_{t}\right\}_{t \geqslant 0}$ is strongly continuous. This completes the proof of the theorem.

As a corollary we obtain a result of Dr. D. J. Ralph [19, Theorem 2.3.2]. This corollary is the analogue (for well-bounded operators of type (B)) of the Hille-Sz. Nagy theorem for selfadjoint operators [14, Theorem 22.3.1].

(4.14) COROLlaRY. Let $\left\{T_{t}\right\}_{t \geqslant 0}$ be a strongly continuous semigroup in $9(X)$, with each $T_{t}$ a well-bounded operator of type $(\mathrm{B})$ whose spectral family is $F_{t}(\cdot)$. Then

(i) for $t \geqslant 0, \sigma\left(T_{t}\right) \geqslant 0$, and $T_{t}$ is one-to-one;

(ii) $\sup \left\{\left\|F_{t}(\lambda)\right\|: t \geqslant 0, \lambda \in \mathbf{R}\right\}<+\infty$;

(iii) there is a unique spectral family $P(\cdot)$ such that for some constant $b \in \mathbf{R}$, $P(\lambda)=I$ for $\lambda \geqslant b$, and

$$
T_{t} x=\lim _{a \rightarrow+\infty} \int_{-a}^{a} e^{\lambda t} d P(\lambda) x, \quad t \geqslant 0, x \in X ;
$$

(iv) the infinitesimal generator $Q$ of $\left\{T_{t}\right\}_{t \geqslant 0}$, with domain $\mathscr{D}(\mathcal{Q})$, is given by

$$
\begin{aligned}
\mathscr{D}(\mathscr{Q}) & =\left\{x \in X: \lim _{a \rightarrow+\infty} \int_{-a}^{a} \lambda d P(\lambda) x \text { exists }\right\}, \\
\mathscr{Q} x & =\lim _{a \rightarrow+\infty} \int_{-a}^{a} \lambda d P(\lambda) x, \text { for } x \in \mathscr{D}(\mathbb{Q}) ;
\end{aligned}
$$

(v) an operator $S \in \Re(X)$ commutes with $T_{t}$ for all $t \geqslant 0$ if and only if $S$ commutes with $P(\lambda)$ for all $\lambda \in \mathbf{R}$.

Conversely, if $P(\cdot)$ is a spectral family such that for some constant $b \in \mathbf{R}, P(\lambda)=I$ for $\lambda \geqslant b$, then the expression for $T_{t}, t \geqslant 0$, in (iii) above defines a strongly continuous semigroup of well-bounded operators of type (B).

Proof. Since $T_{t}=T_{t / 2}^{2}$, it is clear that $\sigma\left(T_{t}\right) \geqslant 0$ for $t \geqslant 0$. Thus each $T_{t}$ is essentially already expressed in terms of its canonical decomposition with $R_{t}=T_{t}$, $A_{t}=0$. By Theorem (4.5) we have that (4.14)(i) holds and for $t>0$,

$$
T_{t}=\int_{[0, \beta]}^{\oplus} \lambda^{t} d F_{1}(\lambda), \quad \text { where } \beta=\max \sigma\left(T_{1}\right)
$$


Conclusion (4.14)(ii) follows from Lemma (4.3) and (4.15). If we define $P(\cdot)$ : $\mathbf{R} \rightarrow \Re(X)$ by setting

$$
P(\lambda)=F_{1}\left(e^{\lambda}\right), \quad \lambda \in \mathbf{R},
$$

then it is easy to see that $P(\cdot)$ is a spectral family such that $P(\lambda)=I$ for $\lambda \geqslant \log \beta$, and that (4.14)(iii) holds for this particular spectral family. If $Q(\cdot)$ is any spectral family satisfying (4.14)(iii), then define $H(\cdot): \mathbf{R} \rightarrow \mathscr{B}(X)$ by setting

$$
H(\lambda)= \begin{cases}Q(\log \lambda), & \text { for } \lambda>0 \\ 0, & \text { for } \lambda \leqslant 0\end{cases}
$$

It is obvious that $H(\cdot)$ is a spectral family concentrated on $\left[0, e^{b}\right]$, and that $T_{1}=\int_{\left[0, e^{b}\right]}^{\oplus} \lambda d H(\lambda)$. Thus $H(\cdot)=F_{1}(\cdot)$ on $\mathbf{R}$, and so $Q(\cdot)$ is the particular spectral family $P(\cdot)$ defined in (4.16). This proves (4.14)(iii). The conclusion (4.14)(v) now follows from (4.16) and (4.14)(iii).

We now take up (4.14)(iv), beginning with the observation that if $\mu>\log \beta$, then [10, Theorem VIII.1.11] $\mu \in \rho(\mathbb{Q})$ and

$$
R(\mu, \mathbb{Q}) x=\int_{0}^{+\infty} e^{-\mu t} T_{t} x d t, \text { for } x \in X .
$$

We fix a number $\mu>\log \beta$. It is easy to see that $\int_{-a}^{\log \beta}(\mu-\lambda)^{-1} d P(\lambda)$ converges in the uniform operator topology, as $a \rightarrow+\infty$, to some operator $M \in \Re(X)$. Suppose $\lambda_{0}<\log \beta$ and $x=\left[I-P\left(\lambda_{0}\right)\right] x$. For $t \geqslant 0, T_{t} x=\int_{\lambda_{0}}^{\log \beta} e^{\lambda t} d P(\lambda) x$. Application of an arbitrary $\phi \in X^{*}$ to this equation and an integration by parts give

$$
\left\langle T_{t} x, \phi\right\rangle=\beta^{t}\langle x, \phi\rangle-\int_{\lambda_{0}}^{\log \beta}\langle P(\lambda) x, \phi\rangle t e^{\lambda t} d \lambda .
$$

Apply $\phi$ to (4.17), substitute (4.18), and interchange the order of integration to get

$$
\langle R(\mu, \mathcal{Q}) x, \phi\rangle=\langle x, \phi\rangle(\mu-\log \beta)^{-1}-\int_{\lambda_{0}}^{\log \beta}\langle P(\lambda) x, \phi\rangle(\mu-\lambda)^{-2} d \lambda .
$$

Another integration by parts shows that $\langle R(\mu, \mathbb{Q}) x, \phi\rangle=\langle M x, \phi\rangle$. Hence

$$
R(\mu, \mathbb{Q})=M \text {. }
$$

If $y \in \mathscr{D}(\mathbb{Q})$, let $z=(\mu-\mathbb{Q}) y$. So $y=R(\mu, \mathbb{Q}) z=M z$. For $u>|\log \beta|$, use the definition of $M$ to get

$$
\int_{-u}^{u}(\mu-\lambda) d P(\lambda) y=\left[\int_{-u}^{\log \beta}(\mu-\lambda) d P(\lambda)\right] M z=[I-P(-u)] z .
$$

Letting $u \rightarrow+\infty$, we see that $\lim _{u \rightarrow+\infty} \int_{-u}^{u} \lambda d P(\lambda) y$ exists and

$$
\mu y-\lim _{u \rightarrow+\infty} \int_{-u}^{u} \lambda d P(\lambda) y=z=\mu y-Q \mathcal{Q} y .
$$

So $\lim _{u \rightarrow+\infty} \int_{-u}^{u} \lambda d P(\lambda) y=Q y$. On the other hand, if $x \in X$ and $\lim _{u \rightarrow+\infty} \int_{-u}^{u} \lambda d P(\lambda) x=w$ exists, then $\lim _{u \rightarrow+\infty} \int_{-u}^{\log \beta}(\mu-\lambda) d P(\lambda) x=\mu x-w$. Using the definition of $M$ and (4.19) we have $x=M(\mu x-w)=[R(\mu, \mathbb{Q})](\mu x-w)$. So $x \in \mathscr{D}(\mathscr{Q})$, and (4.14)(iv) is demonstrated.

To prove the final assertion of the corollary, suppose $P(\cdot)$ is any spectral family such that for some $b \in \mathbf{R}, P(\lambda)=I$ for $\lambda \geqslant b$. Let $H(\cdot)$ be the spectral family given 
by $H(\lambda)=P(\log \lambda)$ for $\lambda>0$ and $H(\lambda)=0$ for $\lambda \leqslant 0$. It is easy to see that for $t>0$,

$$
\lim _{a \rightarrow+\infty} \int_{-a}^{a} e^{\lambda t} d P(\lambda) x=\int_{\left[0, e^{b}\right]}^{\oplus} \mu^{t} d H(\mu) x \quad \text { for } x \in X .
$$

Thus (4.14)(iii) defines a family $\left\{T_{t}\right\}_{t \geqslant 0}$ such that $T_{t}=\int_{\left[0, e^{b}\right]}^{\oplus} \mu^{t} d H(\mu)$ for $t>0$ and $T_{0}=I$. It is evident from this latter characterization that $\left\{T_{t}\right\}_{t \geqslant 0}$ has the required properties.

We now turn our attention to the semigroup $\left\{e^{i A t}\right\}_{t \geq 0}$ in Theorem (4.5). Notice that if we define $A_{-t}=-A_{t}+2 \pi I$ for $t>0$, then by [14, Theorem 16.3.6] $\left\{e^{i A_{t}}\right\}_{t \in \mathbf{R}}$ is a strongly continuous group. Moreover, for $t \in \mathbf{R}, A_{t}$ is well-bounded of type (B) and $\sigma\left(A_{t}\right) \subseteq[0,2 \pi]$. The spectral families of the operators $A_{t}, t \in \mathbf{R}$, are easily seen to be uniformly bounded. We are thus led to seek an analogue for the present context of Stone's theorem [14, Theorem 22.4.3] on one-parameter unitary groups. This goal is accomplished in Theorem (4.20) below. It should be noted, however, that the validity of an analogue is by no means self-evident. The present context and that of Stone's theorem are quite different, in part because in the general setting spectral families are not associated with projection-valued measures, but are, roughly speaking, involved with conditional convergence (as illustrated in the example concerning $L^{p}(\mathbf{T})$ at the end of this section). In particular, the analogue of the integral representation for a unitary group is not a priori guaranteed to be meaningful in the present context, since no projection-valued measure is involved. As will also be seen, the proof of the generalization of Stone's theorem requires more machinery than Corollary (4.14).

Throughout the rest of this paper, a convergent series of the form $\sum_{n=-\infty}^{\infty} u_{n}$ will signify the sum $\left[\sum_{n=0}^{\infty} u_{n}+\sum_{n=1}^{\infty} u_{-n}\right]$, where each of the series inside the brackets converges.

(4.20) THEOREM. Let $\left\{T_{t}\right\}_{t \in \mathbf{R}}$ be a strongly continuous one-parameter group of operators on $X$ with infinitesimal generator $\mathcal{G}$. Suppose that $\left\{T_{t}\right\}_{t \in \mathbf{R}}$ satisfies the following two conditions: (a) for each $t \in \mathbf{R}, T_{t}=e^{i A_{t}}$, where $A_{t}$ is a well-bounded operator of type (B) such that $\sigma\left(A_{t}\right) \subseteq[0,2 \pi]$; and (b) $\sup \left\{\left\|E_{t}(\lambda)\right\|: t, \lambda \in \mathbf{R}\right\} \equiv K$ $<+\infty$, where $E_{t}(\cdot)$ is the spectral family of $A_{t}$, for $t \in \mathbf{R}$. Then

(i) There is a unique spectral family $\mathcal{E}(\cdot)$ for $X$ such that

$$
T_{t} x=\lim _{u \rightarrow+\infty} \int_{-u}^{u} e^{i t \lambda} d \mathcal{E}(\lambda) x, \text { for } t \in \mathbf{R}, x \in X .
$$

(ii) $\left\{T_{t}: t \in \mathbf{R}\right\}^{\prime}=\{\mathcal{E}(\lambda): \lambda \in \mathbf{R}\}^{\prime}$.

(iii) The domain of $\mathcal{G}, \mathscr{D}(\mathcal{G})$, equals

$$
\left\{x \in X: \lim _{u \rightarrow+\infty} \int_{-u}^{u} \lambda d \mathcal{E}(\lambda) x \text { exists }\right\},
$$

and

$$
\mathcal{G}(x)=i \lim _{u \rightarrow+\infty} \int_{-u}^{u} \lambda d \mathcal{E}(\lambda) x \quad \text { for } x \in \mathscr{D}(\mathcal{G})
$$


Proof. By virtue of Proposition (3.13) we shall assume, without loss of generality, that $2 \pi \notin \sigma_{p}\left(A_{t}\right)$ for $t \in \mathbf{R}$; so $T_{t}=e^{i A_{t}}$ is now the canonical decomposition of $T_{t}$, and $E_{t}\left(2 \pi^{-}\right)=I$, for $t \in \mathbf{R}$. Also, $\left\{T_{t}: t \in \mathbf{R}\right\} \cup\left\{A_{t}: t \in \mathbf{R}\right\} \cup\left\{E_{t}(\lambda): t, \lambda \in \mathbf{R}\right\}$ is a commutative family. Let $k$ be a positive integer. The projections

$$
Q_{k, j}=E_{1 / k}\left[\left(2 \pi \frac{j+1}{k}\right)^{-}\right]-E_{1 / k}\left[\left(2 \pi \frac{j}{k}\right)^{-}\right], \quad \text { for } j=0,1, \ldots, k-1
$$

have sum equal to $I$, and satisfy $Q_{k, i} Q_{k, j}=0$ for $i \neq j$. Let $X_{k, j}=Q_{k, j} X$, for $j=0, \ldots, k-1$. Let $\tilde{A}_{k}=\bigoplus_{j=0}^{k-1}\left(k A_{1 / \tilde{z}^{k}}-2 \pi j\right) \mid X_{k, j}$. It is easy to see that $\tilde{A}_{k}$ is a well-bounded operator of type (B), $\sigma\left(\tilde{A}_{k}\right) \subseteq[0,2 \pi]$, and

$$
\exp \left(i \tilde{A}_{k}\right)=\exp \left(i k A_{1 / k}\right)=T_{1 / k}^{k}=T_{1}=\exp \left(i A_{1}\right) .
$$

We now show that $2 \pi \notin \sigma_{p}\left(\tilde{A}_{k}\right)$. Suppose $\tilde{A}_{k} x=2 \pi x$. Let $x_{j}=Q_{k, j} x$ for $j=$ $0, \ldots, k-1$. Then $\sum_{j=0}^{k-1}\left(k A_{1 / k}-2 \pi j\right) x_{j}=\sum_{j=0}^{k-1} 2 \pi x_{j}$. Thus for each $j, A_{1 / k} x_{j}=$ $2 \pi(j+1) k^{-1} x_{j}$. So $x_{j}=\left\{E_{1 / k}[2 \pi(j+1) / k]-E_{1 / k}\left[(2 \pi(j+1) / k)^{-}\right]\right\} x_{j}$. Apply $Q_{k, j}$ to both sides of this equation to get $x_{j}=0$. By (4.21) and Proposition (3.15), $\tilde{A}_{k}=A_{1}$, or, equivalently

$$
A_{1 / k}-k^{-1} A_{1}=\sum_{j=1}^{k-1} 2 \pi j k^{-1}\left\{E_{1 / k}\left[\left(2 \pi \frac{j+1}{k}\right)^{-}\right]-E_{1 / k}\left[\left(2 \pi \frac{j}{k}\right)^{-}\right]\right\}
$$

for each positive integer $k$.

For each $t \in \mathbf{R}$, let $U_{t}=T_{t} e^{-i t A_{1}}$ (cf. Nagy's work reproduced in [20, pp. 381-383]). It is obvious that $\left\{U_{t}\right\}_{t \in \mathbf{R}}$ is a strongly continuous group of operators on $X$ with $U_{1}=I$. The plan of the proof is to obtain a description of the periodic group $\left\{U_{t}\right\}_{t \in \mathbf{R}}$ which will enable us to reach the required conclusions about $\left\{T_{t}\right\}_{t \in \mathbf{R}}$. The periodicity of $\left\{U_{t}\right\}_{t \in \mathbf{R}}$ gives us certain facts about $(C, 1)$-summability automatically (see, e.g., [14, proof of Theorem 16.7.2]). Specifically, for each $n \in \mathbf{Z}$, let $P_{n}=\int_{0}^{1} e^{-2 \pi i n t} U_{t} d t$, the integral being taken as the strong limit of Riemann sums. It is straightforward to see that

$$
U_{s} P_{n}=e^{2 \pi i n s} P_{n} \text { for } s \in \mathbf{R} \text { and } n \in \mathbf{Z} .
$$

Hence from the definition of $\left\{P_{n}\right\}_{n=-\infty}^{\infty}$, each $P_{n}$ is a projection, and $P_{n} P_{m}=0$ for $n \neq m$. From the periodicity and strong continuity of $\left\{U_{t}\right\}_{t \in \mathbf{R}}$, coupled with the standard facts about the Fejér kernel we have

$$
U_{t}=(C, 1) \text {-sum of } \sum_{n=-\infty}^{\infty} e^{2 \pi i n t} P_{n} \text { in the strong operator topology, }
$$

for each $t \in \mathbf{R}$.

In particular, from the definition of $\left\{P_{n}\right\}_{n=-\infty}^{\infty}$ and (4.24), we have

$$
\left\{U_{t}: t \in \mathbf{R}\right\}^{\prime}=\left\{P_{n}: n \in \mathbf{Z}\right\}^{\prime} \text {. }
$$

Our first goal beyond the above standard facts about $\left\{U_{t}\right\}_{t \in \mathbf{R}}$ is to show that in the present setting the series on the right in (4.24) converges in the strong operator topology. For convenience we shall write $E^{(k)}(\cdot)$ instead of $E_{1 / 2^{k}}(\cdot)$ for each positive 
integer $k$. From (4.22) we have

$$
U_{1 / 2^{k}}=\sum_{j=0}^{2^{k}-1} e^{2 \pi i j / 2^{k}}\left\{E^{(k)}\left[\left(2 \pi \frac{j+1}{2^{k}}\right)^{-}\right]-E^{(k)}\left[\left(2 \pi \frac{j}{2^{k}}\right)^{-}\right]\right\} .
$$

Now let $n$ be a fixed but arbitrary nonnegative integer. Let $k$ be an integer such that $2^{k}-1>n$. If

$$
x \in\left\{E^{(k+1)}\left[\left(2 \pi \frac{n+1}{2^{k+1}}\right)^{-}\right]-E^{(k+1)}\left[\left(2 \pi \frac{n}{2^{k+1}}\right)^{-}\right]\right\} X
$$

then by (4.26), $\left(U_{1 / 2^{k+1}}\right)(x)=e^{2 \pi i n / 2^{k+1}} x$. So $\left(U_{1 / 2^{k}}\right) x=\left(U_{1 / 2^{k+1}}\right)^{2} x=e^{2 \pi i n / 2^{k}} x$. Thus, using (4.26) again, we have that

$$
x \in\left\{E^{(k)}\left[\left(2 \pi \frac{n+1}{2^{k}}\right)^{-}\right]-E^{(k)}\left[\left(2 \pi \frac{n}{2^{k}}\right)^{-}\right]\right\} X .
$$

Thus, writing $H_{n, k}$ for $\left\{E^{(k)}\left[\left(2 \pi(n+1) / 2^{k}\right)^{-}\right]-E^{(k)}\left[\left(2 \pi n / 2^{k}\right)^{-}\right]\right\}$, we have

$$
H_{n, k+1} X \subseteq H_{n, k} X \text { for } 2^{k}-1>n \geqslant 0 .
$$

Using (4.23) and (4.26) we see that

$$
P_{n} X \subseteq H_{n, k} X \text { for } 2^{k}-1>n .
$$

Suppose $m \in \mathbf{Z}, m \neq n$. Obviously there is some $k_{0}$ such that $2^{k_{0}}-1>n$ and $m-n \notin 2^{k_{0}} \mathbf{Z}$. It follows by (4.23) and (4.26) that $H_{n, k_{0}} P_{m}=0$, and hence by (4.27) that $H_{n, k} P_{m}=0$ for $k \geqslant k_{0}$. Combining this with (4.28) gives

$$
H_{n, k} x \rightarrow P_{n} x \quad \text { as } k \rightarrow+\infty \text { for } x \in X_{0},
$$

where $X_{0}$ is the linear manifold spanned by the subspaces $P_{j} X$ for $j \in \mathbf{Z}$. However, by hypothesis (b) the projections $H_{n, k}$ are uniformly bounded, while (4.24) for $t=0$ shows that $X_{0}$ is dense in $X$. Applying these facts to (4.29) we have that for $N \in \mathbf{Z}$, $N \geqslant 0$,

(4.30) $\sum_{n=0}^{N} P_{n}=\lim _{k \rightarrow+\infty} \sum_{n=0}^{N} H_{n, k}$ in the strong operator topology of $\Re(X)$.

Recalling the definition of $H_{n, k}$, we notice that $\sum_{n=0}^{N} H_{n, k}=E^{(k)}\left[\left(2 \pi(N+1) / 2^{k}\right)^{-}\right]$. Thus, in view of hypothesis (b) and (4.30),

$$
\left\|\sum_{n=0}^{N} P_{n}\right\| \leqslant K \text { for } N \geqslant 0 \text {. }
$$

Since it is obvious that $\sum_{n=0}^{\infty} P_{n} x$ converges for $x \in X_{0}$, and $X_{0}$ is dense in $X$, we get from (4.31) that

$$
\sum_{n=0}^{\infty} P_{n} \text { converges in the strong operator topology of } \mathscr{B}(X) \text {. }
$$

We next prove the strong convergence of $\sum_{n=1}^{\infty} P_{-n}$. Observe that the one-parameter group $\left\{T_{-t}\right\}_{t \in \mathbf{R}}$ satisfies all the hypotheses employed above for $\left\{T_{t}\right\}_{t \in \mathbf{R}}$, and so we obtain, as above, a periodic group $\left\{V_{t}\right\}_{t \in \mathbf{R}}$ with corresponding "Fourier coefficients" 
$\left\{\tilde{P}_{n}\right\}_{n=-\infty}^{\infty}$. For $t \in \mathbf{R}, V_{t}=T_{-t} \exp \left(-i t A_{-1}\right)$. Let

$$
\tilde{A}=\left(-A_{1}+2 \pi\right)\left|\left\{I-E_{1}(0)\right\} X \oplus 0\right| E_{1}(0) X .
$$

It is easy to verify that $\tilde{A}$ is well bounded of type (B), $\sigma(\tilde{A}) \subseteq[0,2 \pi], 2 \pi \notin \sigma_{p}(\tilde{A})$, and $e^{i \tilde{A}}=e^{-i A_{1}}=\left(T_{1}\right)^{-1}=\exp \left(i A_{-1}\right)$. So $A_{-1}=\tilde{A}$, and we have

$$
V_{t}=e^{-2 \pi i t} U_{-t}\left|\left\{I-E_{1}(0)\right\} X \oplus U_{-t}\right| E_{1}(0) X, \quad \text { for } t \in \mathbf{R} .
$$

For $n \in \mathbf{Z}$ and $x \in\left\{I-E_{1}(0)\right\} X$ (resp., $\left.x \in E_{1}(0) X\right)$, we have $\tilde{P}_{n} x=$ $\int_{0}^{1} e^{-2 \pi i n t} V_{t} x d t$, and so, by (4.33), $\tilde{P}_{n} x=P_{-(n+1)} x$ (resp., $\tilde{P}_{n} x=P_{-n} x$ ). Since $\sum_{n=0}^{\infty} \tilde{P}_{n}$ must converge in the strong operator topology of $\mathscr{B}(X)$, by adaptation of the corresponding result for $\left\{U_{t}\right\}_{t \in \mathbf{R}}$ to $\left\{V_{t}\right\}_{t \in \mathbf{R}}$, it follows from what has just been shown that $\sum_{n=1}^{\infty} P_{-n}$ converges in the strong operator topology of $\mathscr{B}(X)$. Combining this fact with (4.32) and, for $t=0,(4.24)$, we get

$$
\sum_{n=-\infty}^{\infty} P_{n} \text { converges in the strong operator topology with sum equal to } I \text {. }
$$

From this and (4.23) we arrive at the following for $t \in \mathbf{R}$,

(4.34) $U_{t}=\sum_{n=-\infty}^{\infty} e^{2 \pi i n t} P_{n}$, the series converging in the strong operator topology.

Let $\mathcal{G}_{0}$ be the infinitesimal generator of $\left\{U_{t}\right\}_{t \in \mathbf{R}}$. It is obvious from (4.34) that for $n \in \mathbf{Z}, P_{n} X \subseteq\left\{x \in \mathscr{D}\left(\mathcal{G}_{0}\right): \mathcal{G}_{0} x=2 \pi i n x\right\}$. Thus, if $x \in X$ and $\sum_{n=-\infty}^{\infty} n P_{n} x$ converges, then, since $\mathcal{G}_{0}$ is a closed operator, $x \in \mathscr{D}\left(\mathcal{G}_{0}\right)$ and $\mathcal{G}_{0} x=\sum_{n=-\infty}^{\infty} 2 \pi i n P_{n} x$. Conversely, if $x \in \mathscr{D}\left(\mathscr{S}_{0}\right)$, let $\lambda$ be a positive real number, and set $y=\left(\lambda-\mathcal{G}_{0}\right) x$. By [10, Theorem VIII.1.11], $\lambda \in \rho\left(\mathcal{G}_{0}\right)$, and $R\left(\lambda, \mathcal{G}_{0}\right) P_{n} y=\int_{0}^{+\infty} e^{-\lambda t} U_{t} P_{n} y d t$, for all $n \in \mathbf{Z}$, where $R\left(\cdot, \mathcal{G}_{0}\right)$, denotes the resolvent of $\mathcal{G}_{0}$. Using (4.23) in the integral, we obtain $R\left(\lambda, \mathcal{G}_{0}\right) P_{n} y=(\lambda-2 \pi i n)^{-1} P_{n} y$. Thus

$$
x=R\left(\lambda, \mathcal{G}_{0}\right) y=\sum_{n=-\infty}^{\infty}(\lambda-2 \pi i n)^{-1} P_{n} y,
$$

and so for all $n \in \mathbf{Z}, P_{n} x=(\lambda-2 \pi i n)^{-1} P_{n} y$. Hence $\sum_{n=-\infty}^{\infty} n P_{n} x$ converges. We have established

$$
\begin{aligned}
\mathscr{D}\left(\mathcal{G}_{0}\right) & =\left\{x \in X: \sum_{n=-\infty}^{\infty} n P_{n} x \text { converges }\right\}, \text { and } \\
\mathcal{G}_{0} x & =\sum_{n=-\infty}^{\infty} 2 \pi i n P_{n} x, \quad \text { for } x \in \mathscr{D}\left(\mathcal{G}_{0}\right) .
\end{aligned}
$$

As an immediate consequence of (4.35) we have

$$
P_{n} X=\left\{x \in \mathscr{D}\left(\mathcal{G}_{0}\right): \mathcal{G}_{0} x=2 \pi i n x\right\}, \text { for all } n \in \mathbf{Z} .
$$

We now return to consideration of the group $\left\{T_{t}\right\}_{t \in \mathbf{R}}$. From the definition of $\left\{U_{t}\right\}_{t \in \mathbf{R}}$, we have for $t \in \mathbf{R}, x \in X$,

$$
T_{t} x=\sum_{n=-\infty}^{\infty} e^{i t A_{1}} e^{2 \pi i n t} P_{n} x
$$


For convenience, define the spectral family $\tilde{E}_{1}(\cdot)$ by setting $\tilde{E}_{1}(\lambda)=E_{1}(2 \pi \lambda)$ for $\lambda \in \mathbf{R}$. Then $\tilde{E}_{1}(\cdot)$ is concentrated on $[0,1]$, and for $t \in \mathbf{R}, e^{i t A_{1}}=\int_{[0,1]}^{\oplus} e^{2 \pi i t \lambda} d \tilde{E}_{1}(\lambda)$. With an obvious change of variable, (4.37) now becomes

$$
T_{t} x=\sum_{n=-\infty}^{\infty}\left[\int_{[n, n+1]}^{\oplus} e^{2 \pi i t \lambda} d \tilde{E}_{1}(\lambda-n)\right] P_{n} x, \quad \text { for } t \in \mathbf{R}, x \in X .
$$

Now for each $\lambda \in \mathbf{R}$, define $M(\lambda)$ by setting

$$
M(\lambda)=\sum_{n=-\infty}^{[\lambda]-1} P_{n}+P_{[\lambda]} \tilde{E}_{1}(\lambda-[\lambda])
$$

where $[\lambda]$ denotes the greatest integer less than or equal to $\lambda$. Since $A_{1} \in$ $\left\{U_{t}: t \in \mathbf{R}\right\}^{\prime}$, it follows from (4.25) that $\left\{\tilde{E}_{1}(\lambda): \lambda \in \mathbf{R}\right\} \cup\left\{P_{n}: n \in \mathbf{Z}\right\}$ is a commutative family. It is now straightforward to check that $M(\cdot)$ is a spectral family. It is also straightforward to check that for $n \in \mathbf{Z}, t \in \mathbf{R}, x \in X$

$$
\begin{aligned}
{\left[\int_{[n, n+1]}^{\oplus} e^{2 \pi i t \lambda} d \tilde{E}_{1}(\lambda-n)\right] P_{n} x=} & e^{2 \pi i t n} P_{n} \tilde{E}_{1}(0) x-e^{2 \pi i t(n+1)} P_{n+1} \tilde{E}_{1}(0) x \\
& +\int_{[n, n+1]} e^{2 \pi i t \lambda} d M(\lambda) x .
\end{aligned}
$$

Since (4.34) shows that $\sum_{n=-\infty}^{\infty} e^{2 \pi i n t} P_{n} \tilde{E}_{1}(0) x=U_{t} \tilde{E}_{1}(0) x$, substitution of (4.39) in (4.38) gives

$$
T_{t} x=\sum_{n=-\infty}^{\infty} \int_{[n, n+1]} e^{2 \pi i t \lambda} d M(\lambda) x, \text { for } t \in \mathbf{R}, x \in X .
$$

So

$$
T_{t} x=\lim _{n \rightarrow+\infty} \int_{[-n, n]} e^{2 \pi i t \lambda} d M(\lambda) x, \quad \text { for } t \in \mathbf{R}, x \in X .
$$

For $u>0, M(u) \int_{[u]}^{[u]+1} e^{2 \pi i t \lambda} d M(\lambda) x=\int_{[u]}^{u} e^{2 \pi i t \lambda} d M(\lambda) x$, and it follows from the convergence of the series in (4.40) and the uniform boundedness of $M(\cdot)$ that $\int_{[u]}^{u} e^{2 \pi i t \lambda} d M(\lambda) x \rightarrow 0$ as $u \rightarrow+\infty$. Thus, using (4.40) again we see that $\lim _{u \rightarrow+\infty} \int_{0}^{u} e^{2 \pi i t \lambda} d M(\lambda) x$ exists. In a similar fashion we see that $\lim _{u \rightarrow+\infty} \int_{-u}^{0} e^{2 \pi i t \lambda} d M(\lambda) x$ exists. So we have

$$
T_{t} x=\lim _{u \rightarrow+\infty} \int_{-u}^{u} e^{2 \pi i t \lambda} d M(\lambda) x, \text { for } t \in \mathbf{R}, x \in X .
$$

Next, we observe that if $S \in\left\{T_{t}: t \in \mathbf{R}\right\}^{\prime}$, then, in particular, $S$ commutes with $A_{1}$, and so with $U_{t}$, for all $t \in \mathbf{R}$. Hence

$$
\left\{T_{t}: t \in \mathbf{R}\right\}^{\prime} \subseteq\left\{P_{n}: n \in \mathbf{Z}\right\}^{\prime} \cap\left\{\tilde{E}_{1}(\lambda): \lambda \in \mathbf{R}\right\}^{\prime} \subseteq\{M(\lambda): \lambda \in \mathbf{R}\}^{\prime} .
$$

This, in conjunction with $(4.41)$, gives

$$
\left\{T_{t}: t \in \mathbf{R}\right\}^{\prime}=\{M(\lambda): \lambda \in \mathbf{R}\}^{\prime} .
$$

Suppose that $J(\cdot)$ is a spectral family such that $(4.41)$ holds with $J(\cdot)$ in place of $M(\cdot)$. We show that $J(\cdot)=M(\cdot)$. Obviously $\left\{T_{t}: t \in \mathbf{R}\right\} \cup\{J(\lambda): \lambda \in \mathbf{R}\}$ is a 
commutative family; hence by (4.42) so is the union of this family with $\{M(\lambda)$ : $\lambda \in \mathbf{R}$ \}. Let $u$ be an arbitrary positive number, temporarily fixed, and let $X_{u}=$ $[M(u)-M(-u)][J(u)-J(-u)] X$. Clearly $X_{u}$ is invariant under $J(\cdot)$ and $M(\cdot)$. Denote their respective restrictions to $X_{u}$ by $\tilde{J}(\cdot)$ and $\tilde{M}(\cdot)$. It is easy to see that $\tilde{J}(\cdot)$ and $\tilde{M}(\cdot)$ are spectral families for $X_{u}$ such that $\tilde{M}(\lambda)=\tilde{J}(\lambda)=I \mid X_{u}$ for $\lambda \geqslant u$, and $\tilde{M}(\lambda)=\tilde{J}(\lambda)=0 \mid X_{u}$ for $\lambda \leqslant-u$. For $t \in \mathbf{R}$ and $x \in X_{u}$, the hypothesis on $J(\cdot)$ and (4.41) give

$$
T_{t} x=\int_{-u}^{u} e^{2 \pi i t \lambda} d \tilde{M}(\lambda) x=\int_{-u}^{u} e^{2 \pi i t \lambda} d \tilde{J}(\lambda) x
$$

Thus for all $t \in \mathbf{R}$,

$$
\exp \left\{2 \pi i t \int_{[-u, u]}^{\oplus} \lambda d \tilde{M}(\lambda)\right\}=\exp \left\{2 \pi i t \int_{[-u, u]}^{\oplus} \lambda d \tilde{J}(\lambda)\right\} .
$$

Hence $\int_{[-u, u]}^{\oplus} \lambda d \tilde{M}(\lambda)=\int_{[-u, u]}^{\oplus} \lambda d \tilde{J}(\lambda)$, and so $\tilde{M}(\cdot)=\tilde{J}(\cdot)$. Since

$$
[M(u)-M(-u)][J(u)-J(-u)] \rightarrow I
$$

in the strong operator topology of $\Re(X), M(\cdot)=J(\cdot)$.

It is easy to see from the definition of the group $\left\{U_{t}\right\}_{t \in \mathbf{R}}$ that

$$
\mathscr{D}(\mathcal{G})=\mathscr{D}\left(\mathcal{G}_{0}\right) \text { and } \mathcal{G} x=\mathcal{G}_{0} x+i A_{1} x \text { for } x \in \mathscr{D}(\mathcal{G}) \text {. }
$$

Also, it is readily verified from the definition of $M(\cdot)$ that for all $n \in \mathbf{Z}$

$$
\int_{[n, n+1]} \lambda d M(\lambda)=\left[\int_{[n, n+1]} \lambda d \tilde{E}_{1}(\lambda-n)\right] P_{n}+(n+1) P_{n+1} \tilde{E}_{1}(0) .
$$

Since $\tilde{E}_{1}(\lambda)=E_{1}(2 \pi \lambda)$ for $\lambda \in \mathbf{R}$, this last equation can be rewritten as

$$
\int_{[n, n+1]} \lambda d M(\lambda)=(2 \pi)^{-1} A_{1} P_{n}+n P_{n}\left\{I-E_{1}(0)\right\}+(n+1) P_{n+1} E_{1}(0) .
$$

Thus, for $x \in X$ and $N \in \mathbf{Z}$ with $N>0$,

$$
\int_{-N}^{N} \lambda d M(\lambda) x=\sum_{n=-N}^{N-1}\left[(2 \pi)^{-1} A_{1} P_{n}+n P_{n}\left\{I-E_{1}(0)\right\}+(n+1) P_{n+1} E_{1}(0)\right] x .
$$

Observe that $A_{1}=\sum_{n=-\infty}^{\infty} A_{1} P_{n}$ in the strong operator topology. If the left-hand side of (4.44) has a limit as $N \rightarrow+\infty$ (for fixed $x$ ), then so does its image under $E_{1}(0)$; hence using (4.44) we see that $\sum_{n=-\infty}^{\infty} n P_{n} x$ is convergent. Conversely, if $\sum_{n=-\infty}^{\infty} n P_{n} x$ is convergent, then (4.44) shows that $\lim _{N \rightarrow+\infty} \int_{-N}^{N} \lambda d M(\lambda) x=(2 \pi)^{-1} A_{1} x+$ $\sum_{n=-\infty}^{\infty} n P_{n} x$. Combining these facts with (4.35) and (4.43), we have

$$
\begin{array}{r}
\mathscr{D}(\mathcal{G})=\left\{x \in X: \lim _{N \rightarrow+\infty} \int_{-N}^{N} \lambda d M(\lambda) x \text { exists }\right\}, \text { and } \\
\mathcal{G}(x)=\lim _{N \rightarrow+\infty} \int_{-N}^{N} 2 \pi i \lambda d M(\lambda) x, \text { for } x \in \mathscr{D}(\mathcal{G}) .
\end{array}
$$

We can replace the discrete variable $N$ by a continuous variable $u>0$ throughout (4.45) by reasoning analogous to that used in obtaining (4.41) from its discrete 
version. The proof of Theorem (4.20) is now complete upon setting $\mathscr{E}(\lambda)=$ $M(\lambda /(2 \pi))$ for $\lambda \in \mathbf{R}$.

Definition. If $\left\{T_{t}\right\}_{t \in \mathbf{R}}$ is a group satisfying the hypotheses of Theorem (4.20), the unique spectral family $\mathcal{E}(\cdot)$ of $(4.20)(i)$ will be called the Stone-type spectral family of the group.

(4.46) COROLlaRY. Let $\left\{T_{t}\right\}_{t \in \mathbf{R}}$ and $\mathcal{G}$ be as in the hypotheses of Theorem (4.20), and suppose further that $T_{2 \pi}=I$. Then

(i) there is a unique sequence of projections $\left\{P_{n}\right\}_{n=-\infty}^{\infty} \subseteq \Re(X)$ such that

(a) $P_{m} P_{n}=0$ for $m \neq n$, and

(b) for each $t \in \mathbf{R}, T_{t}=\sum_{n=-\infty}^{\infty} e^{i n t} P_{n}$, the series converging in the strong operator topology.

(ii) The Stone-type spectral family $\mathcal{E}(\cdot)$ of the present group $\left\{T_{t}\right\}_{t \in \mathbf{R}}$ is determined by $\mathcal{E}(\lambda)=\sum_{n=-\infty}^{[\lambda]} P_{n}$ for all $\lambda \in \mathbf{R}$.

(iii) $\mathcal{D}(\mathcal{G})=\left\{x \in X: \sum_{n=-\infty}^{\infty} n P_{n} x\right.$ converges $\}$, and $\mathcal{G} x=\sum_{n=-\infty}^{\infty}$ in $P_{n} x$ for $x \in \mathcal{Q}(\mathcal{G})$; hence, in particular, for each $n \in \mathbf{Z}$,

$$
P_{n} X=\{x \in \mathscr{Q}(\mathcal{G}): \mathcal{G} x=\operatorname{in} x\} .
$$

(iv) $\sigma(\mathcal{G})=\sigma_{p}(\mathcal{G})=\left\{\right.$ in: $n \in \mathbf{Z}$ and $\left.P_{n} \neq 0\right\}$.

(v) $\left\{T_{t}: t \in \mathbf{R}\right\}^{\prime}=\left\{P_{n}: n \in \mathbf{Z}\right\}^{\prime}$.

Proof. Most of this corollary has been demonstrated in the course of proving Theorem (4.20). As in the proof of Theorem (4.20), we can assume without loss of generality that $2 \pi \notin \sigma_{p}\left(A_{t}\right)$ for $t \in \mathbf{R}$. For $t \in \mathbf{R}$, let $\tilde{T}_{t}=T_{2 \pi t}$. Then the group $\left\{\tilde{T}_{t}\right\}_{t \in \mathbf{R}}$ has all the properties which the given group in Theorem (4.20) enjoyed throughout the proof there. Also $\tilde{T}_{1}=I$, and so the group $\left\{U_{t}\right\}_{t \in \mathbf{R}}$ corresponding to $\left\{\tilde{T}_{t}\right\}_{t \in \mathbf{R}}$ is just the group $\left\{\tilde{T}_{t}\right\}_{t \in \mathbf{R}}$ itself. Thus, we automatically have a sequence $\left\{P_{n}\right\}_{n=-\infty}^{\infty}$ of projections satisfying parts (a) and (b) of (4.46)(i). It is easy to see that for any sequence $\left\{Q_{n}\right\}_{n=-\infty}^{\infty}$ of projections satisfying (a) and (b) of (4.46)(i), the function $J(\cdot): \mathbf{R} \rightarrow \Re_{(X)}$ given by $J(\lambda)=\sum_{n=-\infty}^{[\lambda]} Q_{n}$ is the Stone-type spectral family of the present group $\left\{T_{t}\right\}_{t \in \mathbf{R}}$. This settles the uniqueness requirement of (4.46)(i), and also proves (4.46)(ii). The conclusion (4.46)(iii) follows by applying (4.35) to the group $\left\{\tilde{T}_{t}\right\}_{t \in \mathbf{R}}$, while (4.46)(v) is obtained by applying (4.25) to $\left\{\tilde{T}_{t}\right\}_{t \in \mathbf{R}}$.

It remains to show (4.46)(iv). Since $\exp \{2 \pi \sigma(\mathcal{G})\} \subseteq\{1\}$ [14, Theorem 16.7.1], $\sigma(\mathcal{G}) \subseteq i \mathbf{Z}$. Suppose $n_{0} \in \mathbf{Z}$ and $P_{n_{0}}=0$. With the aid of summation by parts, it is easy to see that $\sum_{n=-\infty}^{\prime \infty}\left(n_{0}-n\right)^{-1} P_{n}$ converges in the uniform operator topology, where the prime superscript indicates that the index $n_{0}$ is omitted from the summation. If $y$ is any vector in $X$, then $y_{N}=\sum_{n=-N}^{\prime N}-i\left(n_{0}-n\right)^{-1} P_{n} y$ converges to a vector $z$ as $N \rightarrow+\infty$, while (by (4.46)(iii)), $\left(i n_{0}-\mathcal{G}\right) y_{N}=\sum_{n=-N}^{\prime N} P_{n} y \rightarrow y$. Since $\mathcal{G}$ is a closed operator, $\left(i n_{0}-\mathcal{G}\right) z=y$. Since it is straightforward to see that $\left(i n_{0}-\mathcal{G}\right)$ is one-to-one, $i n_{0} \in \rho(\mathcal{G})$. Conclusion (4.46)(iv) is now apparent.

(4.47) EXAMPLES. We close this section with some realizations of its contents from classical analysis. The tools needed to show that our examples satisfy the hypotheses of the abstract theorems are on a par with the classical results which ensue. Thus, our object is strictly to illustrate the kinds of classical phenomena which fall under the results of this section, not to offer abstract proofs of classical phenomena. 
As might be surmised by the central role of (4.34) in the proof of Theorem (4.20), an underlying classical notion of that theorem is Fourier inversion. This will be illustrated in examples (4.47)(i), (ii) below. In order to see that the hypotheses of Theorem (4.20) are satisfied in the examples, we shall need the following proposition [12, Theorem 1 and its proof].

Proposition. Let $G$ be a locally compact abelian group, let $x \in G$, and suppose that $1<p<\infty$. Let $T_{x}$ be the translation operator on $L^{p}(G)$ corresponding to $x$. Then there is a well-bounded operator of type (B), $A_{x}$, with spectral family $E_{x}(\cdot)$, such that $T_{x}=e^{i A_{x}}, \sigma\left(A_{x}\right) \subseteq[0,2 \pi], 2 \pi \notin \sigma_{p}\left(A_{x}\right)$, and $\sup \left\{\left\|E_{x}(\lambda)\right\|: \lambda \in \mathbf{R}\right\} \leqslant K_{p}$, where $K_{p}$ is a constant depending only on $p$, and not on $x$ or $G$. Moreover, $A_{x}$ is a multiplier operator.

REMARK. If, in the above proposition, $x$ has infinite order in $G$, and $p \neq 2$, then the operator $A_{x}$ is not a spectral operator [12, Theorem 3].

EXAMPLE (i). The translation group in $L^{p}(\mathbf{T})$. For $t \in \mathbf{R}$, let $T_{t}$ be the translation operator on $X=L^{p}(\mathbf{T}), 1<p<\infty$, corresponding to $e^{i t}$. The infinitesimal generator $\mathcal{G}$ of $\left\{T_{t}\right\}_{t \in \mathbf{R}}$ is given by $\mathscr{D}(\mathcal{G})=\left\{f \in \mathrm{AC}[0,2 \pi]: f(0)=f(2 \pi)\right.$, and $\left.f^{\prime} \in L^{p}(\mathbf{T})\right\}$, and $\mathcal{G} f=f^{\prime}$ for $f \in \mathscr{D}(\mathcal{G})$ (see, e.g., [7, Example 1.5.5]). Thus, in the notation of Corollary (4.46), $P_{n} X$ is the linear span of the vector $x_{n}=e^{i n(\cdot)}$, for $n \in \mathbf{Z}$ by (4.46)(iii). Part (b) of (4.46)(i), for $t=0$, now gives the M. Riesz decomposition $X=H^{p} \oplus \overline{H_{0}^{p}}\left[16\right.$, pp. 150-154], and shows that $\left\{x_{n}\right\}_{n=-\infty}^{\infty}$ is a conditional basis for $X$. The failure of these phenomena to occur for $p=1$ is mirrored in the fact that if $x$ is an element of infinite order in $\mathbf{T}$, the corresponding translation operator on $L^{1}(\mathbf{T})$ does not have a logarithm in $\mathscr{B}\left(L^{1}(\mathbf{T})\right)$ [13, Theorem 13].

EXAMPle (ii). The translation group in $L^{p}(\mathbf{R})$. For $t \in \mathbf{R}$, let $T_{t}$ be the translation operator on $X=L^{p}(\mathbf{R}), 1<p<\infty$, corresponding to $t$. We shall determine the Stone-type spectral family of $\left\{T_{t}\right\}_{t \in \mathbf{R}}$. A theorem of M. Riesz [11, p. 104] asserts that for each $\lambda \in \mathbf{R}$, the characteristic function of $(-\infty, \lambda]$ is an $L^{p}$-multiplier, and the corresponding multiplier operators $E(\lambda)$ for $\lambda \in \mathbf{R}$ are uniformly bounded. Here we regard $E(\lambda)$ as being defined on all of $L^{p}(\mathbf{R})$ (rather than on $L^{2}(\mathbf{R}) \cap L^{p}(\mathbf{R})$ ). It is obvious that $E(\lambda)$ is a projection, and that (2.2)(ii) is satisfied. To see that (iii) and (iv) of (2.2) are satisfied, we observe that

$$
E(\lambda) f=2^{-1}\left\{f-i \chi_{\lambda} H\left(\chi_{-\lambda} f\right)\right\} \quad \text { for } f \in X, \lambda \in \mathbf{R},
$$

where $\chi_{\lambda}(x)=e^{i \lambda x}$ for $x \in \mathbf{R}$, and $H$ is the Hilbert transform on $X=L^{p}(\mathbf{R})$ (see, e.g. [11, pp. 104, 105 and Theorem 6.7.4]). It is obvious from this representation for $E(\cdot)$ that $E(\cdot)$ is continuous on $\mathbf{R}$ in the strong operator topology of $\mathscr{B}(X)$. Thus to show that $E(\cdot)$ is a spectral family for $X$ we need only verify $(2.2)(\mathrm{v})$. For $g \in L^{2}(\mathbf{R})$ we denote its Fourier transform by $\hat{g}$. Let $a$ be an arbitrary positive real number, and let $f \in L^{2}(\mathbf{R}) \cap X$. Then

$$
\{E(a)-E(-a)\} f=(2 \pi)^{-1 / 2} \int_{-a}^{a} \hat{f}(y) e^{i(\cdot) y} d y .
$$

A close examination of the proof on pp. 770-772 of [15] shows that the right-hand side of this equation tends to $f\left[\operatorname{mean}^{p}\right]$ as $a \rightarrow+\infty$. Since

$$
\{E(a)-E(-a)\} f \rightarrow f \text { as } a \rightarrow+\infty,
$$


we can apply $E(0)$ to get $\{E(0)-E(-a)\} f \rightarrow E(0) f$. It follows that $E(\lambda) f \rightarrow 0$ as $\lambda \rightarrow-\infty$. Using this in (4.49) gives $E(\lambda) f \rightarrow f$ as $\lambda \rightarrow+\infty$. From the uniform boundedness of $E(\cdot)$ and the fact that $L^{2}(\mathbf{R}) \cap X$ is dense in $X,(2.2)(\mathrm{v})$ now follows, and so $E(\cdot)$ is a spectral family. We now verify that (4.20)(i) holds for the spectral family $E(\cdot)$. If $t \in \mathbf{R}, f \in L^{2}(\mathbf{R}) \cap X$, and $u>0$, then it is straightforward to see from Fourier transforms that for almost all $x \in \mathbf{R}$,

$$
\left\{\int_{-u}^{u} e^{i t \lambda} d E(\lambda) f\right\}(x)=(2 \pi)^{-1 / 2} \int_{-u}^{u} \hat{f}(y) e^{i(t+x) y} d y .
$$

The right-hand side of this, as a function of $x$, is by equation (4.48) equal to $T_{t}\{E(u)-E(-u)\} f$. Thus the left-hand side of $(4.50)$ tends to $T_{t} f$ [mean $^{p}$ ] as $u \rightarrow+\infty$. From

$$
\left\|\int_{-u}^{u} e^{i t \lambda} d E(\lambda) f\right\|_{p}=\left\|T_{t}\{E(u)-E(-u)\} f\right\|_{p},
$$

it follows that

$$
\left\|\int_{-u}^{u} e^{i t \lambda} d E(\lambda)\right\| \leqslant 2 \sup \{\|E(\lambda)\|: \lambda \in \mathbf{R}\}
$$

for $t \in \mathbf{R}$ and $u>0$. Combining this uniform boundedness result with the fact that, for $f \in L^{2}(\mathbf{R}) \cap X$, the left-hand side of (4.50) tends to $T_{t} f\left[\right.$ mean $\left.^{p}\right]$ as $u \rightarrow+\infty$, we see that $E(\cdot)$ is the Stone-type spectral family of $\left\{T_{t}\right\}_{t \in \mathbf{R}}$.

EXAMPLE (iii). Second order linear differential operators with constant coefficients in $L^{p}(\mathbf{R})$. Let $X$ and $\left\{T_{t}\right\}_{t \in \mathbf{R}}$ be as in Example (ii). Let $\mathrm{AC}_{\text {loc }}$ be the set of all locally absolutely continuous complex-valued functions on $\mathbf{R}$. We write $D$ for the differentiation operator in $X$, i.e., $\mathscr{D}(D)=\left\{f \in X: f \in \mathrm{AC}_{\mathrm{loc}}\right.$ and $\left.f^{\prime} \in X\right\}$, and $D f=f^{\prime}$ for $f \in \mathscr{D}(D)$. The operator $D$ is the infinitesimal generator of $\left\{T_{t}\right\}_{t \in \mathbf{R}}$ [7, Theorem 1.3.13]. We denote the Gauss-Weierstrass operators by $W_{t}, t \geqslant 0$. Thus $W_{0}=I$, and

$$
\left[W_{t} f\right](x)=(4 \pi t)^{-1 / 2} \int_{\mathbf{R}} f(x-u) e^{-u^{2} / 4 t} d u,
$$

for $t>0, f \in X, x \in \mathbf{R}$. It is known that $\left\{W_{t}\right\}_{t \geqslant 0}$ is a strongly continuous semigroup whose infinitesimal generator is $D^{2}$ (see [7, Theorem 4.3.11]). It is shown in [19, Theorem 3.3.2] that $W_{t}$ is well-bounded for $t \geqslant 0$ (since $X$ is reflexive, each $W_{t}$ is also of type (B)). Thus by, for instance, (4.14)(i), $\sigma\left(W_{t}\right) \geqslant 0$ and $W_{t}$ is one-to-one for $t \geqslant 0$. Now let $b, c$ be real constants, and for $t \geqslant 0$ define $S_{t} \in \Re_{(}(X)$ by setting $S_{t}=$ $e^{c t} W_{t} T_{b t}$. Then $\left\{S_{t}\right\}_{t \geqslant 0}$ is a strongly continuous semigroup of polar operators and the definition gives the canonical decomposition of $S_{t}$. Thus $\left\{S_{t}\right\}_{t \geqslant 0}$ satisfies the hypotheses of Theorem (4.5). We show that the infinitesimal generator $\mathcal{Q}$ of $\left\{S_{t}\right\}_{t \geqslant 0}$ is

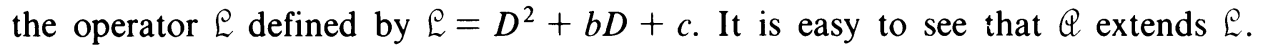
However, by [10, Theorems VII.9.7 and VII.9.10], $\varrho$ is a closed operator, and $\sigma(\mathcal{L}) \subseteq\{z \in \mathbf{C}: \operatorname{Re} z \leqslant c\}$. In particular, $\rho(\mathscr{Q})$ and $\rho(\mathcal{L})$ intersect, and it follows,

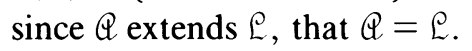

5. Semigroups and the spectral theory of ordinary differential operators. This section has two purposes: first to illustrate the preceding abstract theory of wellbounded operators and polar operators with a substantial set of classical ordinary 
differential operators, and second to apply the functional calculus of well-bounded operators to the further study of differential operators.

Let $n \geqslant 1$ denote a fixed integer, and let $a_{j}, 0 \leqslant j \leqslant n-2$, denote functions in $L^{\infty}(-\pi, \pi)$. For sufficiently differentiable functions $u$ on $(-\pi, \pi)$, a differential expression is defined by

$$
\tau(u)=(-i)^{n} u^{(n)}+a_{n-2}(x) u^{(n-2)}+\cdots+a_{0}(x) u .
$$

Boundary value expressions are defined by

$$
U_{j}(u)=\sum_{k=0}^{n-1}\left[a_{j k} u^{(k)}(-\pi)+b_{j k} u^{(k)}(\pi)\right], \quad 1 \leqslant j \leqslant n,
$$

where $a_{j k}, b_{j k}$ are complex constants. A closed, densely defined linear operator $L$ is defined on each space $L^{p}(-\pi, \pi), 1<p<\infty$, with domain $D(L)$ consisting of all functions $u$ in $C^{n-1}[-\pi, \pi]$ such that $u^{(n-1)}$ is absolutely continuous, $u^{(n)}$ is in $L^{p}(-\pi, \pi)$, and $U_{j}(u)=0,1 \leqslant j \leqslant n$. For $u$ in $D(L)$, we have

$$
L u=\tau(u)
$$

The order of the boundary value expression $U_{j}$ is the largest integer $k$ such that $\left|a_{j k}\right|+\left|b_{j k}\right|>0$. Denote the order of $U_{j}$ by $k_{j}$. By performing linear operations on the original $U_{j}$ 's, it is possible to assume, without loss of generality, that the boundary conditions are in normalized form

$$
U_{j}(u)=a_{j} u^{\left(k_{j}\right)}(-\pi)+b_{j} u^{\left(k_{j}\right)}(\pi)+\sum_{k=0}^{k_{j}-1}\left[a_{j k} u^{(k)}(-\pi)+b_{j k} u^{(k)}(\pi)\right],
$$

where $n-1 \geqslant k_{1} \geqslant k_{2} \geqslant \cdots \geqslant k_{n} \geqslant 0$ and $k_{j+2}<k_{j}$.

Let $\omega_{1}, \omega_{2}, \ldots, \omega_{n}$ denote the distinct $n$th roots of -1 , labeled so that $\operatorname{Re}\left(\omega_{1}\right) \leqslant$ $\operatorname{Re}\left(\omega_{2}\right) \leqslant \cdots \leqslant \operatorname{Re}\left(\omega_{n}\right)$.

(5.5) Definition. If $n$ is odd; $n=2 \mu-1$, the normalized boundary expressions (5.4) are Birkhoff regular if the numbers $\theta_{0}, \theta_{1}$, defined by the identity

$$
\theta_{0}+\theta_{1} s=\left|\begin{array}{ccc}
a_{1} \omega_{1}^{k_{1}} \cdots a_{1} \omega_{\mu-1}^{k_{1}} & \left(a_{1}+s b_{1}\right) \omega_{\mu}^{k_{1}} & b_{1} \omega_{\mu+1}^{k_{1}} \cdots b_{1} \omega_{n}^{k_{1}} \\
a_{2} \omega_{1}^{k_{2}} \cdots a_{2} \omega_{\mu-1}^{k_{2}} & \left(a_{2}+s b_{2}\right) \omega_{\mu}^{k_{2}} & b_{2} \omega_{\mu+1}^{k_{2}} \cdots b_{2} \omega_{n}^{k_{2}} \\
\vdots & \vdots & \vdots \\
a_{n} \omega^{k_{n}} \cdots a_{n} \omega_{\mu-1}^{k_{n}} & \left(a_{n}+s b_{n}\right) \omega_{\mu}^{k_{n}} & b_{n} \omega_{\mu+1}^{k_{n}} \cdots b_{n} \omega_{n}^{k_{n}}
\end{array}\right|
$$

are not zero.

If $n$ is even; $n=2 \mu$, the normalized boundary expressions (5.4) are Birkhoff regular if the numbers $\theta_{-1}, \theta_{1}$, defined by the identity

$\theta_{-1} / s+\theta_{0}+\theta_{1} s$

$$
=\left|\begin{array}{cccc}
a_{1} \omega_{1}^{k_{1}} \cdots a_{1} \omega_{\mu-1}^{k_{1}} & \left(a_{1}+s b_{1}\right) \omega_{\mu}^{k_{1}} & \left(a_{1}+\frac{1}{s} b_{1}\right) \omega_{\mu+1}^{k_{1}} & b_{1} \omega_{\mu+2}^{k_{1}} \cdots b_{1} \omega_{n}^{k_{1}} \\
a_{2} \omega_{2}^{k_{2}} \cdots a_{2} \omega_{\mu-1}^{k_{2}} & \left(a_{2}+s b_{2}\right) \omega_{\mu}^{k_{2}} & \left(a_{2}+\frac{1}{s} b_{2}\right) \omega_{\mu+1}^{k_{2}} & b_{2} \omega_{\mu+2}^{k_{2}} \cdots b_{2} \omega_{n}^{k_{2}} \\
\vdots & \vdots & \vdots & \vdots \\
a_{n} \omega_{n}^{k_{n}} \cdots a_{n} \omega_{\mu-1}^{k_{n}} & \left(a_{n}+s b_{n}\right) \omega_{\mu}^{k_{n}} & \left(a_{n}+\frac{1}{s} b_{n}\right) \omega_{\mu+1}^{k_{n}} & b_{n} \omega_{\mu+2}^{k_{n}} \cdots b_{n} \omega_{n}^{k_{n}}
\end{array}\right|
$$

are not zero. 
Further, the boundary expressions (5.4) are Birkhoff regular with simple spectrum if either (i) $n$ is odd or (ii) $n$ is even and $\theta_{0}^{2}-4 \theta_{-1} \theta_{1} \neq 0$.

This definition was given in [6]. An exposition can be found in [18, pp. 48-55]. The definition of Birkhoff regularity fails to identify intrinsic spectral theoretic properties of linear operators; however, based on classical asymptotic analysis of the eigenvalues and eigenfunctions of such operators, it is known that the eigenfunction expansions associated with these operators have the essential properties of ordinary Fourier series, both with respect to pointwise convergence $[6,25,3]$ and convergence in the norms of the Banach spaces $L^{p}(-\pi, \pi), 1<p<\infty[2,3]$. One of our results is that the resolvent operator $R(\lambda, L)$ of any Birkhoff regular operator with simple spectrum is a compact well-bounded operator, for any $\lambda$ in the resolvent set of $L$, thereby placing the $L^{p}$-norm results on eigenfunction expansions for these operators in a spectral theoretic context.

Returning to an abstract setting, let $L$ denote a closed, densely defined linear operator on a reflexive Banach space $X$, let $\lambda$ be a point in the resolvent set of $L$, and let $R(\lambda, L)$ be the resolvent operator of $L$ at $\lambda$. Suppose $R(\lambda, L)$ is well-bounded. We show that the functional calculus can be extended to give an expression for $L$ in terms of the spectral decomposition for $R(\lambda, L)$, and if $L$ generates a strongly continuous semigroup, then the semigroup also has a representation using the functional calculus on $R(\lambda, L)$.

Let $C$ be a simple, nonclosed rectifiable arc in the complex plane containing the spectrum of $R(\lambda, L)$. We shall assume that $L$ is unbounded, so that zero is in $C$. Let $S$ denote the arc length of $C$, and let $\sigma:[0, S] \rightarrow C$ denote the arc length parameterization of $C$. Let $s_{0}=\sigma^{-1}(0)$. For $\alpha>0$, let

$$
C(\alpha)=\left\{\mu \in C:\left|\sigma^{-1}(\mu)-s_{0}\right| \geqslant \alpha\right\} .
$$

Let $E(\mu), \mu \in C$, denote the spectral decomposition of $R(\lambda, L)$,

$$
R(\lambda, L)=\int_{C}^{\oplus} \mu d E(\mu) .
$$

(5.6) Lemma. For each $f$ in $X, \lim _{s \rightarrow s_{0}^{-}} E(\mu(s)) f=E(0) f$.

Proof. From Proposition 2.5, $E(0)-E\left(\mu\left(0^{-}\right)\right)$is a projection on the eigenmanifold of $R(\lambda, L)$ corresponding to eigenvalue $\mu=0$. Since 0 is not an eigenvalue of a resolvent, $E(0)=E\left(\mu\left(0^{-}\right)\right)$.

Let $P(\alpha)$ denote the projection operator defined by $P(\alpha)=\int_{C(\alpha)}^{\oplus} d E(\mu)$.

(5.7) Lemma. For each $f$ in $X, \lim _{\alpha \rightarrow 0} P(\alpha) f=f$.

Proof. We have $P(\alpha)=E\left(\mu\left(s_{0}-\alpha\right)\right)+I-E\left(\mu\left(s_{0}+\alpha\right)\right)$. From the definition of a spectral family, $E(\mu(s))$ is continuous from above, and by the previous lemma, $E(\mu(s))$ is continuous from below at $s_{0}$. Thus, in the strong operator topology,

$$
E\left(\mu\left(s_{0}^{-}\right)\right)=E\left(\mu\left(s_{0}^{+}\right)\right) \text {. }
$$

(5.8) CoROllaRY. The vectors of the form $P(\alpha) g$, for $g$ in $X$ and $\alpha>0$, form a dense set in $X$. 
For any function $F(\mu)$ of bounded variation on $C$, let

$$
\begin{gathered}
\||F|\|=\max _{C}|F(\mu)|+\underset{C}{\operatorname{var} F,} \\
F_{\alpha}= \begin{cases}F, & \mu \text { in } C(\alpha), \\
0, & \mu \text { in } C-C(\alpha) \quad(\alpha>0) .\end{cases}
\end{gathered}
$$

Let $K(t, \mu)$ be a complex-valued function, defined for $\mu$ in $C$ and for $0 \leqslant t \leqslant t_{0}$, some $t_{0}>0$.

(5.11) THEOREM. Assume

(i) $\|K(t, \cdot)\| \leqslant M_{0}<\infty, 0 \leqslant t \leqslant t_{0}$,

(ii) for each $\alpha>0$,

$$
\lim _{t \rightarrow 0^{+}}\left\|\left|(K(t, \cdot)-1)_{\alpha}\right|\right\|=0
$$

Then for each $f$ in $D(L)$,

$$
\lim _{t \rightarrow 0^{+}} \lim _{\alpha \rightarrow 0^{+}} \int_{C(\alpha)}^{\oplus} K(t, \mu) \frac{1}{\mu} d E(\mu) f=(\lambda I-L) f .
$$

Proof. We use the functional calculus for well-bounded operators, which extends to functions of bounded variation on reflexive Banach spaces [9, p. 354]. Since $K_{\alpha}(t, \mu)_{\mu}^{\frac{1}{\mu}}$ is in $\operatorname{BV}(C)$, there is a bounded linear operator $L_{\alpha}(t)$ given by $L_{\alpha}(t)=$ $\int_{C}^{\oplus} K_{\alpha}(t, \mu) \frac{1}{\mu} d E(\mu)$. If $f$ is in $D(L)$, there exists $g$ in $X$ such that $f=R(\lambda, L) g$,

$$
f=\int_{C}^{\oplus} \mu d E(\mu) g \text {. }
$$

Using the functional calculus, for $\operatorname{such} f$,

$$
L_{\alpha}(t) f=L_{\alpha}(t) R(\lambda, L) g=\int_{C}^{\oplus} K_{\alpha}(t, \mu) d E(\mu) g .
$$

Define operators

$$
\Lambda_{\alpha}(t)=\int_{C}^{\oplus} K_{\alpha}(t, \mu) d E(\mu), \quad L(t)=\int_{C}^{\oplus} K(t, \mu) d E(\mu) .
$$

Since $\left\|K_{\alpha}(t, \cdot)\right\| \leqslant 2\|K(t, \cdot)\| \leqslant 2 M_{0}$, we see that there is a uniform bound on $\left\|\Lambda_{\alpha}(t)\right\|$ for $\alpha \geqslant 0,0 \leqslant t \leqslant t_{0}$. If $g=P_{\beta} g$ for some $\beta>0$, then

$$
L(t) g=\Lambda_{\beta}(t) g=\Lambda_{\alpha}(t) g, \quad \alpha \leqslant \beta,
$$

so for fixed $t, 0<t \leqslant t_{0}$, and all $g$ in $X, \lim _{\alpha \rightarrow 0^{+}} \Lambda_{\alpha}(t) g=L(t) g$. Thus if $f$ is in $D(L), g=(\lambda I-L) f$,

$$
\lim _{\alpha \rightarrow 0^{+}} \int_{C}^{\oplus} K_{\alpha}(t, \mu) \frac{1}{\mu} d E(\mu) f=\int_{C}^{\oplus} K(t, \mu) d E(\mu) g .
$$

Since $\|K(t, \cdot)-1\|$ is bounded as $t \rightarrow 0^{+}$, there is a uniform bound for $\|L(t)-I\|$ as $t \rightarrow 0^{+}$. If $g=P_{\beta} g$, then

$$
(L(t)-I) g=\int_{C}^{\oplus}(K(t, \mu)-1)_{\beta} d E(\mu) g,
$$

and by condition (ii), $(L(t)-I) g \rightarrow 0$ as $t \rightarrow 0^{+}$for such $g$. Since these $g$ 's are dense in $X$, this is true for all $g$ in $X$, so if $g=(\lambda I-L) f$, we have (5.12). 
(5.13) COROllary. If $f$ is in $D(L)$,

$$
\lim _{\alpha \rightarrow 0^{+}} \int_{C(\alpha)}^{\oplus} \frac{1}{\mu} d E(\mu) f=(\lambda I-L) f .
$$

Proof. Let $K(t, \mu) \equiv 1$.

(5.14) Theorem. Assume $C$ is such that, for some $t_{0}>0$, there exists $M>0$ such that

(1) $\left|\left\|e^{t / \mu}-1\right\|\right| M, 0<t \leqslant t_{0}$,

(2) $\left\|\left(e^{t / \mu}-1\right) /(t / \mu)\right\| \leqslant M, 0<t \leqslant t_{0}$,

(3) $\lim _{t \rightarrow 0^{+}}\left\|\left(e^{t / \mu}-1\right)_{\alpha}\right\| \|=0, \alpha>0$,

(4) $\lim _{t \rightarrow 0^{+}}\left\|\left(\left(e^{t / \mu}-1\right) /(t-\mu)-1\right)_{\alpha}\right\| \|=0, \alpha>0$.

Then $-L$ generates a strongly continuous semigroup $U(t)$ on $X$, and

$$
U(t)=e^{-\lambda t} \int_{C}^{\oplus} e^{t / \mu} d E(\mu) .
$$

Proof. Since $e^{t / \mu}$ is of bounded variation, we use the functional calculus to see that $W(t)=\int_{C}^{\oplus} e^{t / \mu} d E(\mu)$ is a semigroup of bounded linear operators. We show that $W(t)$ is strongly continuous at $t=0$, and that the infinitesimal generator of $W(t)$ is $\lambda I-L$.

We have $W(t)-I=\int_{C}^{\oplus}\left(e^{t / \mu}-1\right) d E(\mu)$. Let

$$
(W(t)-I)_{\alpha}=\int_{C}^{\oplus}\left(e^{t / \mu}-1\right)_{\alpha} d E(\mu) .
$$

Now $\|W(t)-I\|$ is uniformly bounded, $0 \leqslant t \leqslant t_{0}$, by (1) and the functional calculus. If $g=P_{\beta} g$ for some $\beta>0$, then

$$
(W(t)-I) g=(W(t)-I)_{\alpha} g, \quad \alpha \leqslant \beta,
$$

and since, by (3), $\left\|(W(t)-I)_{\alpha}\right\| \rightarrow 0$ as $t \rightarrow 0$, we see that $W(t) g \rightarrow g$ as $t \rightarrow 0$, on a dense set of $g$ 's. By the uniform boundedness, this holds for all $f$ in $X$, establishing strong continuity.

For any $f$ in $X$,

$$
\frac{W(t)-I}{t} f=\int_{C}^{\oplus} \frac{e^{t / \mu}-1}{t / \mu} \frac{1}{\mu} d E(\mu) f .
$$

Let $K(t, \mu)=\left(e^{t / \mu}-1\right) /(t / \mu)$. Using conditions (2) and (4), we see that the hypotheses of Theorem (5.11) are satisfied. Thus for $f$ in $D(L)$,

$$
\lim _{t \rightarrow 0^{+}} \frac{W(t)-I}{t} f=(\lambda I-L) f .
$$

Strictly speaking, this shows that the infinitesimal generator of $W(t)$ is an extension of $\lambda I-L$; but since $\lambda I-L$ has an everywhere defined bounded inverse, the extension is $\lambda I-L$.

(5.15) TheOREM. Suppose $R(\lambda, L)$ is well bounded on an arc $C$, where $\lambda$ is a real number such that $\lambda<\operatorname{Re}(\sigma(L))$. Let

$$
F_{\nu}(\mu)=\frac{\mu}{1+\nu \mu} \quad(\nu<0)
$$


If $C$ is such that there exists a constant $M>0$, so that for each integer $r \geqslant 0$,

$$
\underset{C}{\operatorname{var}} F_{\nu}^{r} \leqslant \frac{M}{|\nu|^{r}}
$$

then $-L$ generates a strongly continuous semigroup.

Proof. Since $\operatorname{Re}(\sigma(\lambda I-L))<0$, we have $\operatorname{Re} \sigma(R(\lambda, L)) \leqslant 0$. If $s$ is in the resolvent set of $L$, we have the resolvent equation

$$
R(s)-R(\lambda)=(\lambda-s) R(\lambda) R(s), \quad(R(s)=R(s, L))
$$

or

$$
R(s)=R(\lambda)[I+(s-\lambda) R(\lambda)]^{-1} .
$$

Since $R(\lambda)$ is well-bounded, we can use the functional calculus on (5.17)

$$
R(s)=\int_{C}^{\oplus} \frac{\mu}{1+(s-\lambda) \mu} d E(\mu),
$$

provided $1+(s-\lambda) \mu \neq 0$ for $\mu$ in $C$. In particular, if $s$ is real, $s<\lambda$, then $\operatorname{Re}(\mu(s-\lambda)) \geqslant 0$, so $1+(s-\lambda) \mu \neq 0$. Let $\nu=s-\lambda$. Again using the functional calculus, for each integer $r \geqslant 0, R^{r}(s)=\int_{C}^{\oplus} F_{\nu}^{r}(\mu) d E(\mu)$, and then

$$
\left\|R^{r}(s)\right\| \leqslant K\left\|F_{\nu}^{r}\right\|=K\left[\max _{C}\left|F_{\nu}^{r}(\mu)\right|+\underset{C}{\operatorname{var}} F_{\nu}^{r}\right],
$$

where $K>0$ is the constant implementing the well-bounded operator $R(\lambda)$. Now

$$
\left|F_{\nu}^{r}(\mu)\right|=\frac{1}{|\nu|^{r}}\left|\frac{\nu \mu}{1+\nu \mu}\right|^{r} \leqslant \frac{1}{|\nu|^{r}},
$$

since $\operatorname{Re} \nu \mu \geqslant 0$. Using this, along with (5.16) in (5.18), we see that there is a constant $M^{\prime}>0$ such that

$$
\left\|R^{r}(s)\right\| \leqslant \frac{M^{\prime}}{|s-\lambda|^{r}}, \quad s<\lambda .
$$

Thus by the Hille-Yosida-Phillips theorem [7, p. 34], $-L$ generates a strongly continuous semigroup.

(5.19) Corollary. If $\sigma(R(\lambda, L))$ is contained in a real interval $C=(-a, 0]$, then the well-boundedness of $R(\lambda, L)$ is sufficient for $-L$ to generate a strongly continuous semigroup.

Proof. As $\mu \rightarrow 0,(\nu \mu /(1+\nu \mu))^{r}$ decreases monotonically to zero, and

$$
(\nu \mu /(1+\nu \mu))^{r}<1 .
$$

Thus (5.16) is satisfied with $M=1$.

We return now to the case that $L$ is a Birkhoff regular differential operator with simple spectrum, and consider asymptotic estimates for the eigenvalues and their reciprocals.

The eigenvalues of $L$ form two sequences $\lambda_{j k}, j=1,2, k \rightarrow+\infty$, with $\lambda_{j k}=\rho_{j k}^{n}$ and

$$
\rho_{j k}=(-1)^{j} k+\alpha_{j}+i \beta_{j}+\frac{\gamma_{j}}{k}+\frac{\delta_{j k}}{k},
$$


where $\alpha_{j}, \beta_{j}$ are real constants, $\gamma_{j}$ is a complex constant, and $\left\{\delta_{j k}\right\}$ is a complex sequence in $l^{2}\left[5\right.$, p. 300]. Additionally, if $n$ is even, then denoting by $\xi_{1}, \xi_{2}$ the distinct roots of $\theta_{1} \xi^{2}+\theta_{0} \xi+\theta_{-1}$, we have

$$
\frac{1}{2 \pi i} \operatorname{Ln} \xi_{j}=\alpha_{j}+i \beta_{j} .
$$

Since $\theta_{-1}= \pm \theta_{1}\left[22\right.$, p. 361], we have $\xi_{1} \xi_{2}= \pm 1$, so that $\beta_{1}=-\beta_{2}$. Thus $\alpha_{1}=\alpha_{2}$ implies $\beta_{1} \neq 0$ and $\beta_{1}=0$ implies $\alpha_{1} \neq \alpha_{2}$. Since $2 \pi \alpha_{1}, 2 \pi \alpha_{2}$ are angles, $-\frac{1}{2}<\alpha_{j} \leqslant \frac{1}{2}$, we have $0 \leqslant \alpha_{1}-\alpha_{2}<1$ (assuming $\alpha_{1} \geqslant \alpha_{2}$ ).

(5.21) LEMMA. Let $n \geqslant 1$ be a fixed integer, and let $\left\{B_{k}\right\}, k \geqslant 1$, be a real bounded sequence. If $n>B_{k}-B_{k+1}$ for $k$ sufficiently large, then the sequence $D_{k}=k^{n}+$ $B_{k} k^{n-1}$ is eventually monotone increasing.

Proof. Using the binomial theorem, the inequality $D_{k+1}>D_{k}$ is equivalent to

$$
\left(n-B_{k}+B_{k+1}\right) k^{n-1}>-\sum_{l=0}^{n-2}\left[\left(\begin{array}{l}
n \\
l
\end{array}\right)+B_{k+1}\left(\begin{array}{c}
n-1 \\
l
\end{array}\right)\right] k^{\prime} .
$$

Since the coefficients of $k^{l}$ on the right are bounded, this inequality holds for $k$ sufficiently large.

(5.22) COROLLARY. With the conditions of the previous lemma, the sequence

$$
A_{k}=\frac{1}{k^{n}}\left[1+\frac{B_{k}}{k}\right]
$$

is eventually monotonically convergent to zero.

Proof. Let $D_{k}=1 / \dot{A}_{k}$. Using the geometric series, we have $D_{k}=k^{n}+B_{k}^{\prime} k^{n-1}$, where $B_{k}^{\prime}=-B_{k}\left(1+B_{k} / k\right)$. Then for $k$ sufficiently large, $n>B_{k}^{\prime}-B_{k+1}^{\prime}$.

For any complex $\lambda$, not equal to any of the $\lambda_{j k}$, let

$$
\mu_{j k}=\left(\lambda-\lambda_{j k}\right)^{-1} \text {. }
$$

The $\mu_{j k}$ are the eigenvalues of $R(\lambda, L)$. Assuming the order $n$ of $L$ is $\geqslant 2$ (the case $n=1$ is easy to consider directly), we have

$$
\begin{aligned}
\lambda_{j k}-\lambda & =(-1)^{j n} k^{n}\left[1+\frac{\alpha_{j}}{k}+i \frac{\beta_{j}}{k}+\frac{\gamma_{j}}{k^{2}}+\frac{\delta_{j k}}{k^{2}}\right]^{n} \\
& =(-1)^{j n} k^{n}\left[1+\frac{n \alpha_{j}}{k}+i \frac{n \beta_{j}}{k}+\frac{\gamma_{j}}{k^{2}}+\frac{\delta_{j k}}{k^{2}}\right]^{n}
\end{aligned}
$$

where the $\gamma_{j}$ and $\delta_{j k}$ are not the same with each occurrence. In (5.23) we use the fact that translation by $\lambda$ affects only $\gamma_{j k}$. From (5.23),

$$
\mu_{j k}=-\frac{(-1)^{j n}}{k^{n}}\left[1-\frac{n \alpha_{j}}{k}-i \frac{n \beta_{j}}{k}+\frac{\gamma_{j}}{k^{2}}+\frac{\delta_{j k}}{k^{2}}\right] .
$$

(5.25) LEMMA. If $n$ is odd, $\left\{\operatorname{Re} \mu_{j k}\right\}$ is eventually monotonically decreasing to zero for $j=1$, and eventually monotonically increasing to zero for $j=2$. If $n$ is even, then for each $j,\left\{\operatorname{Re} \mu_{j k}\right\}$ is eventually monotonically increasing to zero. 
Proof. Using (5.24),

$$
\operatorname{Re} \mu_{j k}=\frac{-(-1)^{j n}}{k^{n}}\left[1-\frac{n \alpha_{j}}{k}+\frac{E_{j k}}{k^{2}}\right]
$$

for some bounded real sequence $E_{j k}$. Suppose $n$ is odd and $j=1$. Then $\left\{\operatorname{Re} \mu_{j k}\right\}$ satisfies the conditions of Corollary (5.22). The other cases are handled similarly.

(5.26) LEMMA. If $n$ is odd and $\beta_{1} \neq 0, \beta_{2} \neq 0$, then

$$
\begin{array}{ll}
\lim _{k \rightarrow \infty} \operatorname{Arg}\left(\lambda_{j k}-\lambda\right)=0 & (j=2), \\
\lim _{k \rightarrow \infty} \operatorname{Arg}\left(\lambda_{j k}-\lambda\right)=\pi & (j=1),
\end{array}
$$

and in both cases the convergence is eventually monotone.

If $n$ is even and $\beta_{1} \neq 0$, then

$$
\lim _{k \rightarrow \infty} \operatorname{Arg}\left(\lambda_{j k}-\lambda\right)=0 \quad(j=1,2),
$$

and again the convergence is eventually monotone for each $j$.

Proof. Assume $n$ is odd and $j=2$. From (5.23),

$$
\frac{\operatorname{Im}\left(\lambda_{2 k}-\lambda\right)}{\operatorname{Re}\left(\lambda_{2 k}-\lambda\right)}=\frac{n \beta_{2} / k+\gamma_{2} / k^{2}+\delta_{2 k} / k^{2}}{1+n \alpha_{2} / k+E_{2 k} / k^{2}}
$$

where $E_{2 k}$ is a bounded real sequence. Since $\beta_{2} \neq 0$,

$$
\frac{\operatorname{Im}\left(\lambda_{2 k}-\lambda\right)}{\operatorname{Re}\left(\lambda_{2 k}-\lambda\right)}=\frac{n \beta_{2}}{k}\left[1+\frac{1}{k}\left(\gamma_{2}+\delta_{2 k}-n \alpha_{2}\right)\right]
$$

where $\delta_{2 k}$ has been redefined, but still $\delta_{2 k} \rightarrow 0$. With $B_{k}=\gamma_{2}-n \alpha_{2}+\delta_{2 k}$, the hypotheses of Corollary (5.22) are satisfied. The other cases are handled similarly.

As a direct consequence of this lemma, we have

(5.27) THEOREM. If $n$ is odd and $\beta_{1} \neq 0, \beta_{2} \neq 0$, then

$$
\begin{aligned}
& \lim _{k \rightarrow \infty} \operatorname{Arg} \mu_{j k}=0, \quad j=1, \\
& \lim _{k \rightarrow \infty} \operatorname{Arg} \mu_{j k}=\pi, \quad j=2,
\end{aligned}
$$

eventually monotonically, while if $n$ is even and $\beta_{1} \neq 0$

$$
\lim _{k \rightarrow \infty} \operatorname{Arg} \mu_{j k}=\pi, \quad j=1,2,
$$

eventually monotonically for each $j$.

(5.28) LemMA. For each $j,\left|\mu_{j k}\right|$ is eventually monotone.

Proof. Using (5.24) and again redefining constants,

$$
\left|\mu_{j k}\right|^{2}=\frac{1}{k^{2 n}}\left[1+\frac{F}{k}+\frac{G_{k}}{k^{2}}\right],
$$

where $G_{k}$ is bounded. This satisfies Corollary (5.22). 
(5.29) LeMmA. Let $\left\{x_{k}\right\},\left\{y_{k}\right\}$ be real sequences, $k=1,2, \ldots$, with $x_{1}>x_{2}>$ $\cdots>x_{k}>\cdots>0, x_{k} \rightarrow 0$, and $\Sigma_{1}^{\infty}\left|y_{k}-y_{k+1}\right|<\infty$. Then there exists a piecewise linear absolutely continuous function $H(x)$ on $\left[0, x_{1}\right]$ such that $H\left(x_{k}\right)=y_{k}$, and the graph of $H$ is rectifiable.

Proof. Define $H$ by $H\left(x_{k}\right)=y_{k}$, linear otherwise. The monotonicity of $\left\{x_{k}\right\}$ permits this. Thus $H$ is differentiable a.e., and

$$
\int_{0}^{x_{1}}\left|H^{\prime}(x)\right| d x=\sum_{1}^{\infty}\left|y_{k}-y_{k+1}\right|<\infty \text {. }
$$

The length of the graph of $H$ is

$$
\sum_{k=1}^{\infty} \sqrt{\left(x_{k}-x_{k+1}\right)^{2}+\left(y_{k}-y_{k+1}\right)^{2}} \leqslant \sum_{1}^{\infty}\left|x_{k}-x_{k+1}\right|+\sum_{1}^{\infty}\left|y_{k}-y_{k+1}\right|<\infty .
$$

(5.30) THEOREM. Let $n>1$ be odd. Then the points $\left\{\mu_{j k}\right\}, j=1,2, k=1,2, \ldots$, lie on a simple, nonclosed rectifiable arc $C$ in the complex plane, passing through the origin.

Proof. For fixed $j$ and $k$ sufficiently large, the construction in Lemma (5.29) can be used. The finitely many points omitted in this process can now be adjoined, and then the union of the two curves for $j=1,2$ gives the arc $C$.

For $n$ odd, $\operatorname{Re} \mu_{2 k}<0<\operatorname{Re} \mu_{1 k}$, providing a convenient separation between the points $\mu_{1 k}, \mu_{2 k}$. When $n$ is even, $\operatorname{Re} \mu_{j k}<0, j=1,2$, making the construction of the arc $C$ somewhat more delicate.

(5.31) LEMMA. If $n$ is even,

$$
\sum_{k=1}^{\infty}\left(\left|\mu_{2 k}-\mu_{1 k}\right|+\left|\mu_{1 k}-\mu_{2, k+1}\right|\right)<\infty .
$$

Proof. Using (5.24), we see that each of these terms is $O\left(k^{-(n+1)}\right)$.

(5.32) LEMMA. If $n$ is even and $\alpha_{1}>\alpha_{2}$, then for $k$ sufficiently large

$$
\operatorname{Re} \mu_{2 k}<\operatorname{Re} \mu_{1 k}<\operatorname{Re} \mu_{2, k+1}<\operatorname{Re} \mu_{1, k+1} .
$$

Proof. As before, we have the inequality $\operatorname{Re} \mu_{j k}<\operatorname{Re} \mu_{j, k+1}$. Consider the possibility that $\operatorname{Re} \mu_{2 k}<\operatorname{Re} \mu_{1 k}$. From the formula in the proof of Lemma (5.25), this is equivalent to

$$
n\left(\alpha_{1}-\alpha_{2}\right)>\frac{E_{2 k}-E_{1 k}}{k}
$$

which is true for $k$ sufficiently large. The inequality $\operatorname{Re} \mu_{1 k}<\operatorname{Re} \mu_{2, k+1}$ is equivalent to

$$
\frac{1}{(k+1)^{n}}\left[1-\frac{n \alpha_{2}}{k+1}+\frac{E_{2 k+1}}{(k+1)^{2}}\right]<\frac{1}{k^{n}}\left[1-\frac{n \alpha_{1}}{k}+\frac{E_{1 k}}{k^{2}}\right] .
$$

Taking reciprocals, replacing $\frac{1}{k+1}$ by $\frac{1}{k}$, redefining the $E_{j k}$ 's, and dividing by $k^{n}$, we have

$$
\left(1+\frac{1}{k}\right)^{n}\left[1+\frac{n \alpha_{2}}{k}+\frac{E_{2 k}}{k^{2}}\right]>1+\frac{n \alpha_{1}}{k}+\frac{E_{1 k}}{k^{2}} .
$$


Using the binomial theorem on $\left(1+\frac{1}{k}\right)^{n}$ and again redefining the $E_{j k}$ 's, this is equivalent to

$$
n\left[1-\left(\alpha_{1}-\alpha_{2}\right)\right]>\frac{E_{1 k}-E_{2 k}}{k} .
$$

Since $\alpha_{1}-\alpha_{2}<1$, this last inequality is valid for $k$ sufficiently large.

Consider the piecewise linear directed curve $\mu_{2 k} \rightarrow \mu_{1 k} \rightarrow \mu_{2, k+1}$. By Lemma (5.31), the union $C$ of these curves for all $k$ has finite length.

(5.33) Theorem. Let $n \geqslant 2$ be even. Then the points $\left\{\mu_{j k}\right\}, j=1,2, k=1,2, \ldots$, lie on a simple, nonclosed rectifiable arc $C$ in the complex plane, with the origin as a limit point.

Proof. It suffices to show that the curve $C$ defined above is free of selfintersection. If $\alpha_{1}>\alpha_{2}$, then the monotonicity stated in Lemma (5.32) guarantees this. If $\alpha_{1}=\alpha_{2}$, then because of the assumption of simple spectrum, $\beta_{1}=-\beta_{2} \neq 0$. Thus the sequence $\left\{\mu_{1 k}\right\}$ is separated from $\left\{\mu_{2 k}\right\}$ by the real axis. We still have $\left\{\operatorname{Re} \mu_{j k}\right\}$ monotone for each $j$, and $\operatorname{Re} \mu_{1 k}<\operatorname{Re} \mu_{2, k+1}$. Using (5.24) with $\alpha_{1}=\alpha_{2}$, $\beta_{1}=-\beta_{2} \neq 0$, we see that the line segment $\mu_{2, k+1} \rightarrow \mu_{1, k+1}$ has slope $m_{k}$, where $\left|m_{k}\right|>a k$ for some $a>0$, whereas the line segment $\mu_{1 k} \rightarrow \mu_{2, k+1}$ has bounded slope, uniformly for $k$ sufficiently large. Thus this last line segment cannot intersect any of the preceding ones. The finitely many points omitted in this construction can now be adjoined.

Having established some facts about the location of the $\mu_{j k}$ 's, it is now convenient to relabel these as $\left\{\mu_{k}\right\},-\infty<k<\infty$. Let $S$ denote the length of the arc $C$, and let $\sigma:[0, S] \rightarrow C$ denote the arc length parameterization of $C$, oriented so that $\sigma^{-1}\left(\mu_{k+1}\right)>\sigma^{-1}\left(\mu_{k}\right)$. Since $0 \in C$, we define

$$
s_{0}=\sigma^{-1}(0), \quad A(s)=\left\{k: \sigma^{-1}\left(\mu_{k}\right) \leqslant s\right\} .
$$

Monotonicity Assumption I. (i) $A\left(s_{0}\right)=\{0,1,2, \ldots\}$,

(ii) there exist integer-valued functions $K_{1}(s), K_{2}(s)$, with $K_{1}$ monotone nondecreasing, $K_{2}$ monotone nonincreasing, such that

$$
\begin{array}{ll}
A(s)=\left\{0,1, \ldots, K_{1}(s)\right\}, & 0 \leqslant s<s_{0}, \\
A(s)=A\left(s_{0}\right) \cup\left\{-K_{2}(s),-K_{2}(s)-1, \ldots\right\}, & s_{0}<s \leqslant S .
\end{array}
$$

Montonicity Assumption II. (i) $s_{0}=S$ and $A\left(s_{0}\right)=\{0, \pm 1, \pm 2, \ldots\}$,

(ii) there exist integer-valued, monotone nondecreasing functions $K_{1}(s), K_{2}(s)$ such that

$$
A(s)=\left\{0,1, \ldots, K_{1}(s)\right\} \cup\left\{-1,-2, \ldots,-K_{2}(s)\right\} .
$$

For Birkhoff regular problems with simple spectrum, Monotonicity Assumption I is satisfied if $n$ is odd, while Monotonicity Assumption II is satisfied if $n$ is even.

Let $\varphi_{k}(x)=e^{i k x}, P_{k} f=\left(f, \varphi_{k}\right) \varphi_{k}$ for any integer $k, x$ in $[-\pi, \pi]$, and $f$ integrable. For a fixed curve $C$, and for $\mu$ in $C$, let

$$
\tilde{E}(\mu) f=\sum P_{k} f, \quad k \in A\left(\sigma^{-1}(\mu)\right) .
$$


(5.35) Lemma. For each space $L^{p}(-\pi, \pi), 1<p<\infty$, there exists a constant $K=K_{p}>0$ such that for $\tilde{E}(\mu): L^{p} \rightarrow L^{p}$,

$$
\|\tilde{E}(\mu)\| \leqslant K, \quad \mu \text { in } C
$$

provided one of the monotonicity assumptions holds.

Proof. Because of the boundedness of the conjugate function mapping [17, pp. 46-49,68] $\Sigma P_{k}$ is uniformly bounded when the sum is taken over any finite consecutive set of integers, as well as over the complements of such sets.

(5.36) Lemma. Any sequence $\left\{\mu_{k}\right\}$ satisfying a monotonicity assumption is of bounded variation.

ProOF. $\Sigma_{-\infty}^{\infty}\left|\mu_{k}-\mu_{k+1}\right| \leqslant \Sigma_{-\infty}^{\infty} \int_{\mu_{k}}^{\mu_{k+1}} d \sigma=S<\infty$.

(5.37) THEOREM. If $\left\{\mu_{k}\right\}$ satisfies a monotonicity assumption, then the linear operator $\tilde{R}: L^{p} \rightarrow L^{p}(1<p<\infty)$ defined by

$$
\tilde{R} f=\sum_{-\infty}^{\infty} \mu_{k} P_{k} f
$$

is a well-bounded operator on C. Furthermore, if $\left\{\left|\mu_{k}\right|\right\}$ and $\left\{\operatorname{Arg} \mu_{k}\right\}$ are eventually monotone, then $\tilde{R}$ is a polar operator.

Proof. Since $\left\{\mu_{k}\right\}$ is of bounded variation, $\left\{\mu_{k}\right\}$ defines a multiplier transform $\tilde{R}$ [11, Theorem 6.3.5] on $L^{p}, 1<p<\infty$. The operators $\tilde{E}(\beta)$ define a decomposition of the identity for $L^{p}$ on $C[19$, Corollary 3.2.6], with the obvious extension to the case of complex multipliers, and the resulting well-bounded operator $\int_{C}^{\oplus} \mu d \tilde{E}(\mu)$ coincides with $\tilde{R}$.

If $\left\{\left|\mu_{k}\right|\right\}$ and $\left\{\operatorname{Arg} \mu_{k}\right\}$ are eventually monotone, then

$$
\tilde{R}_{0}=\sum_{-\infty}^{\infty}\left|\mu_{k}\right| P_{k}, \quad \tilde{A}=\sum_{-\infty}^{\infty}\left(\operatorname{Arg} \mu_{k}\right) P_{k}
$$

define commuting well-bounded operators with $2 \pi$ not an eigenvalue of $\tilde{A}$, and $\tilde{R}=\tilde{R}_{0} e^{i \tilde{A}}$.

(5.39) LEMMA. The property of being well-bounded (polar) is invariant with respect to similarity transforms.

Proof. If $T$ is a well-bounded operator implemented by a constant $K>0$ and a curve $C$, and if $B$ is a bounded operator with bounded inverse, then for any polynomial $p$,

$$
\left\|p\left(B T B^{-1}\right)\right\|=\left\|B p(T) B^{-1}\right\| \leqslant\|B\|\left\|B^{-1}\right\|\|p\|_{C},
$$

see $[21$, p. 634].

If $T=R e^{i A}$, then $B T B^{-1}=B R B^{-1} e^{i B A B^{-1}}$, and $B R B^{-1}$ and $B A B^{-1}$ are still wellbounded by the first part of the proof.

Let $\left\{u_{k}(x)\right\}$ denote the eigenfunctions of the differential operator $L$, and let $\left\{v_{k}(x)\right\}$ denote the eigenfunctions of $L^{*}$, normalized so that

$$
\left(u_{k}, v_{j}\right)=\delta_{k j}, \quad k, j=0, \pm 1, \pm 2, \ldots
$$


(5.40) THEOREM. Let $L$ be a Birkhoff regular operator with simple spectrum. For each $p, 1<p<\infty$, there exists a bounded linear operator $B: L^{p} \rightarrow L^{p}$, with bounded inverse, such that

$$
B \varphi_{k}=u_{k}, \quad B^{-1} \varphi_{k}=v_{k} .
$$

Proof. This is proved in [3, Theorem 4.1, 5.3].

(5.41) THEOREM. Let L be a Birkhoff regular operator with simple spectrum. Let $\lambda$ be in the resolvent set of $L$. Then $R(\lambda, L)$ is a well-bounded operator on $L^{p}(-\pi, \pi)$, $1<p<\infty$. If $\beta_{1} \neq 0, \beta_{2} \neq 0$, then $R(\lambda, L)$ is a polar operator.

Proof. For $f$ in $L^{p}$,

$$
R(\lambda, L) f=\sum_{-\infty}^{\infty} \mu_{k}\left(f, v_{k}\right) u_{k}=B \sum_{-\infty}^{\infty}\left(B^{-1} f, \varphi_{k}\right) \varphi_{k}=B \tilde{R} B^{-1} f
$$

where $\tilde{R}$ satisfies the conditions of Theorem (5.37). If $\beta_{1} \neq 0, \beta_{2} \neq 0$, then $\left|\mu_{k}\right|$, $k \rightarrow+\infty$, and $\left|\mu_{k}\right|, k \rightarrow-\infty$, are eventually monotone and $\operatorname{Arg} \mu_{k}, k \rightarrow+\infty$, Arg $\mu_{k}, k \rightarrow-\infty$, are eventually monotone.

The simplest Birkhoff regular problem occurs for $n=1$,

$$
\tau(u)=-i u^{\prime}, \quad U_{1}(u)=u(-\pi)-u(\pi) .
$$

Its eigenfunctions are $\varphi_{k}(x)=e^{i k x}$, corresponding to eigenvalues $\lambda_{k}=k, k=$ $0, \pm 1, \pm 2, \ldots$. This operator, denoted by $L_{0}$, generates the group of translations in $L^{p}(-\pi, \pi), 1 \leqslant p<\infty\left[7\right.$, p. 66]. Let $R_{0}=\left(-\frac{1}{2}-L_{0}\right)^{-1}$. The spectrum of $R_{0}$ consists of the points

$$
\mu_{k}^{0}=\frac{-2}{1+2 k}, \quad k=0, \pm 1, \pm 2, \ldots,
$$

and is contained in the interval $J_{0}=[-2,2]$. Let $L$ be a Birkhoff regular operator with simple spectrum of any order $n$, and let $\lambda$ be a given point in the resolvent set of $L$. If the points $\left\{\mu_{k}\right\}, k=0, \pm 1, \ldots$, satisfy Monotonicity Assumption I, then a piecewise linear monotone increasing map $\psi: J_{0} \rightarrow[0, S]=J$ is defined by $\psi\left(\mu_{k}^{0}\right)=$ $\sigma^{-1}\left(\mu_{k}\right)$, linear otherwise. Thus $\psi(0)=s_{0}$. If the points $\left\{\mu_{k}\right\}$ satisfy Monotonicity Assumption II, the same definition is used, but because of the interlacing of the $\mu_{k}$ 's, $\psi$ is two-to-one, and $s_{0}=S$. Also, in this case $\psi$ is monotone increasing on $[-2,0]$ and monotone decreasing on $[0,2]$. In either case, $\psi$ is of bounded variation on $J_{0}$.

(5.42) THEOREM. Let $B$ denote the operator of Theorem (5.40). Then

$$
R(\lambda, L)=B\left[(\sigma \circ \psi) R_{0}\right] B^{-1} .
$$

Proof. Since $\psi$ is piecewise monotone and $\sigma$ is absolutely continuous, $\sigma \circ \psi$ is absolutely continuous [26, p. 377], and thus the functional calculus for well-bounded operators applies to $\sigma \circ \psi$. Since $\sigma \circ \psi\left(\mu_{k}^{0}\right)=\mu_{k}$, we see that $(\sigma \circ \psi) R_{0}=\tilde{R}$.

Let $E_{0}(x),-2 \leqslant x \leqslant 2$, denote the spectral family for $R_{0}$,

$$
E_{0}(x)=\sum P_{k}, \quad \mu_{k}^{0} \leqslant x .
$$


Let $J(\mu)=\psi^{-1}\left(\left(0, \sigma^{-1}(\mu)\right]\right)$ and let $X_{\mu}(x)$ be the characteristic function of $J(\mu)$. Then

$$
\tilde{E}(\mu)=\int_{J_{0}}^{\oplus} X_{\mu}(x) d E_{0}(x), \quad E(\mu)=B \tilde{E}(\mu) B^{-1} .
$$

(5.44) THEOREM. Let $L$ be an nth order Birkhoff regular differential operator with simple spectrum. Then $B$ maps $D\left(L_{0}^{n}\right)$ onto $D(L)$, and for all $f$ in $D(L)$,

$$
(\lambda I-L) f=\lim _{\alpha \rightarrow 0^{+}} B \int_{J(\alpha)}^{\oplus} \frac{1}{\sigma(s)} d \tilde{E}(s) B^{-1} f .
$$

Proof. From Theorem 3.1 in [4], we know that $B$ maps $D\left(L_{0}^{n}\right)$ onto $D(L)$. The formula is a consequence of Corollary (5.13).

We now show that if $n$ is even and $\alpha_{1} \neq \alpha_{2}$, then $-L$ generates a strongly continuous semigroup on $L^{p}(-\pi, \pi), 1<p<\infty$, which has the spectral representation given in Theorem (5.14). The requirement that $\alpha_{1} \neq \alpha_{2}$ has the consequence that the arc $C$ containing the points $\mu_{k}$ ultimately has a single-valued projection onto the real axis, and $C$ has bounded slope near the origin.

(5.45) LeMMA. Let $n$ be even and let $L$ be an $n$th order Birkhoff regular differential operator with simple spectrum, such that $\alpha_{1} \neq \alpha_{2}$. Then for $k$ sufficiently large, the slopes of the line segments used in the definition of the arc $C$ are bounded.

Proof. Using (5.24), we see that

$$
\begin{aligned}
& \frac{\operatorname{Im}\left(\mu_{2 k}-\mu_{1 k}\right)}{\operatorname{Re}\left(\mu_{2 k}-\mu_{1 k}\right)}=\frac{1}{\alpha_{2}-\alpha_{1}}\left[\beta_{2}-\beta_{1}+O\left(\frac{1}{k}\right)\right], \\
& \frac{\operatorname{Im}\left(\mu_{2, k+1}-\mu_{1 k}\right)}{\operatorname{Re}\left(\mu_{2, k+1}-\mu_{1 k}\right)}=\frac{1}{1-\left(\alpha_{1}-\alpha_{2}\right)}\left[\beta_{2}-\beta_{1}+O\left(\frac{1}{k}\right)\right] .
\end{aligned}
$$

These denominators are not zero, the first by assumption and the second by the remarks preceding Lemma (5.21). These two ratios represent the slopes of the line segments $\mu_{2 k} \rightarrow \mu_{1 k}$ and $\mu_{1 k} \rightarrow \mu_{2, k+1}$ respectively.

We shall also need the curve obtained from $C$ by the inversion $\mu \rightarrow 1 / \mu$. Let $C=(x, y(x))$ for $X$ sufficiently close to zero.

(5.46) Lemma. There exists a constant $A>0$ such that for $x$ sufficiently close to zero,

$$
\left|\frac{\left[x^{2}+y^{2}\right] y^{\prime}-2 y\left[x+y y^{\prime}\right]}{x^{2}+y^{2}-2 x\left[x+y y^{\prime}\right]}\right| \leqslant A \quad\left(y^{\prime}=\frac{d y}{d x}\right) .
$$

Proof. Using (5.24), we see that there is a constant $M \rightarrow 0$ such that

$$
\left|\frac{\operatorname{Im} \mu_{k}}{\operatorname{Re} \mu_{k}}\right| \leqslant M\left|\operatorname{Re} \mu_{k}\right|^{1 / n}
$$

where $M$ is large enough so that this inequality holds for all points on the straight line joining two consecutive $\mu_{k}$ 's. Thus $|y(x) / x| \leqslant M|x|^{1 / n}$, implying that $\lim _{x \rightarrow 0}|y(x) / x|=0$. Dividing numerator and denominator of (5.47) by $x^{2}$, the resulting fraction has bounded numerator, and the denominator converges to 1 . We use the fact that $y^{\prime}$ is bounded (previous lemma). 
(5.48) Lemma. For the curve $C$ of the previous lemma, there exists $M>0$ such that

$$
\underset{C}{\operatorname{var}} F_{\nu}^{r}(\mu) \leqslant \frac{M}{|\nu|^{r}}, \quad r=1,2, \ldots,
$$

for $\nu<0$.

Proof. Let the variation of $F_{\nu}^{r}$ over $C$ be denoted by

$$
I(r, \nu)=\int_{C}\left|\frac{d}{d \mu} F_{\nu}^{r}(\mu)\right||d \mu| .
$$

With $\mu=x+i y(x)$, let $\tau=1 / \mu$. Then

$$
\tau=u-i v=\frac{x}{x^{2}+y^{2}}-i \frac{y}{x^{2}+y^{2}}, \quad d \mu=-\frac{1}{\tau^{2}} d \tau .
$$

If $\Gamma$ is the image of $C$ under $\mu \rightarrow \tau$, we have

$$
I(r, \nu)=\int_{\Gamma} \frac{r}{|\tau+\nu|^{r+1}}|d \tau| .
$$

Since $y=y(x)$ for $x$ close to zero, we have $v=v(u)$ for $u \rightarrow-\infty$, so

$$
|d \tau|=\sqrt{1+(d v / d u)^{2}} d u .
$$

Now

$$
\left|\frac{d v}{d u}\right|=\left|\frac{\left(x^{2}+y^{2}\right) y^{\prime}-2 y\left(x+y y^{\prime}\right)}{\left(x^{2}+y^{2}\right)-2 x\left(x+y y^{\prime}\right)}\right| \leqslant A
$$

by Lemma (5.46). Thus for some $M>0,|d \tau| \leqslant M d u$. Also, $|\tau+\nu| \geqslant|u+\nu|$ $(\nu<0)$. Setting $w=u+\nu$, we have

$$
I(r, \nu) \leqslant \frac{M}{(-1)^{r+1}} \int_{-\infty}^{0} \frac{r}{(u+\nu)^{r+1}} d u=\frac{-M}{(-1)^{r+1}} \int_{-\infty}^{\nu} d u^{-r}=\frac{M}{|\nu|^{r}} .
$$

(5.49) THEOREM. Let $n$ be an even integer, and let $L$ be an nth order Birkhoff regular differential operator with simple spectrum, satisfying the additional condition that $\alpha_{1} \neq \alpha_{2}$. Then $-L$ generates a strongly continuous semigroup $U(t)$ on $L^{p}(-\pi, \pi)$, $1<p<\infty$. Furthermore, let $\lambda$ be a real number such that $\lambda<\operatorname{Re} \sigma(L)$. Let $R(\lambda, L)$ $=\int_{C}^{\oplus} \mu d E(\mu)$. Then the semigroup $U(t)$ has the representation

$$
U(t)=e^{\lambda t} \int_{C}^{\oplus} e^{t / \mu} d E(\mu)
$$

Proof. Using Theorem (5.15) and Lemma (5.48), we see that $-L$ generates a strongly continuous semigroup. To obtain (5.50), we show that the hypotheses of Theorem (5.14) are satisfied.

For $\alpha>0$, let $\Gamma(\alpha)$ denote the image in the $\tau$-plane of the curve $C(\alpha)$, under the transformation $\mu \rightarrow \tau=1 / \mu$. Then $\Gamma(\alpha)$ has a single-valued projection onto the $u$-axis as an interval $u_{1} \leqslant u \leqslant u_{2}<0$. Let $f(\mu)=e^{t / \mu}$ for given $t>0$, and let 
$F(\tau)=e^{t \tau}$. Then

$$
\begin{aligned}
\int_{C(\alpha)}\left|f^{\prime}(\mu)\right||d \mu| & =\int_{\Gamma(\alpha)}\left|F^{\prime}(\tau)\right||d \tau| \leqslant M t \int_{u_{1}}^{u_{2}} e^{u t} d t \\
& =M\left(e^{u_{2} t}-e^{u_{1} t}\right) .
\end{aligned}
$$

This expression is bounded by $M$ for all $t$ and all $-\infty \leqslant u_{1}<u_{2} \leqslant 0$, and converges to zero as $t \rightarrow 0$, when $u_{2}<0$. Combining this with $\left|e^{t / \mu}\right| \leqslant 1, \lim _{t \rightarrow 0^{+}} e^{t / \mu}=1$ for $\mu$ in $C(\alpha)$, we have (1) and (3) of Theorem (5.14).

Now let $f(\mu)=\left(e^{t / \mu}-1\right) /(t / \mu)$ for given $t \geqslant 0$. We see that $f(\mu)$ is bounded on $C$, uniformly in $t \geqslant 0$, and $f(\mu) \rightarrow 1$ as $t \rightarrow 0^{+}$, uniformly on each $C(\alpha)$. Now

$$
F(\tau)=\frac{e^{t \tau}-1}{t \tau}, \quad F^{\prime}(\tau)=t \frac{1-e^{t \tau}(1-t \tau)}{(t \tau)^{2}}
$$

so

$$
\int_{\Gamma(\alpha)}\left|F^{\prime}(\tau)\right||d \tau|=\int_{\Gamma(\alpha)}\left|\frac{1-e^{t \tau}(1-t \tau)}{(t \tau)^{2}}\right||d t \tau| .
$$

Let $s=t \tau=w-i z$, where $w=t u$. Then $|d s| \leqslant M d w$ and

$$
\begin{aligned}
\int_{\Gamma(\alpha)}\left|F^{\prime}(\tau)\right||d \tau| & \leqslant M \int_{w_{1}}^{w_{2}}\left|\frac{1-e^{s}(1-s)}{s^{2}}\right| d w \\
& \leqslant M \int_{w_{1}}^{w_{2}} \frac{\left|1-e^{s}(1-s)\right|}{w^{2}} d w .
\end{aligned}
$$

This last integral is uniformly bounded for $-\infty \leqslant w_{1}<w_{2}<0$, and for $-\infty<w_{1}<$ $w_{2}<0$, converges to zero as $t \rightarrow 0^{+}$. This establishes (2) and (4) of Theorem (5.14).

To show that the operators $U(t)$ in the semigroup are themselves well-bounded operators, we first consider an abstract result on mappings of well-bounded operators.

Let $C$ be an admissible arc in the complex plane, of length $S_{C}$, and let $\rho$ : $\left[0, S_{C}\right] \rightarrow C$ be the arc length parameterization. Let $\alpha=\rho(0), \beta=\rho\left(S_{C}\right)$. Let $f$ be a continuous, complex-valued function which maps $C$ in a one-to-one manner onto an admissible arc $\Gamma$, of length $S_{\Gamma}$. Let $\gamma=f(\alpha), \delta=f(\beta)$, and let $\Gamma$ have the orientation induced by the natural ordering of $\left[0, S_{C}\right]$. Let $\sigma:\left[0, S_{\Gamma}\right] \rightarrow \Gamma$ be the arc length parameterization of $\Gamma$, with $\sigma(0)=\gamma, \sigma\left(S_{\Gamma}\right)=\delta$. Let $h:\left[0, S_{C}\right] \rightarrow\left[0, S_{\Gamma}\right]$ be defined by $y=\sigma^{-1}(f(\rho(x)))=h(x)$. Since $h$ is continuous and strictly monotone, $h^{-1}$ is also continuous, and thus $h, h^{-1}$ are uniformly continuous.

Note that $0=x_{0}<x_{1}<\cdots<x_{n-1}<x_{n}=S_{C}$ is a partition of $\left[0, S_{C}\right]$ if and only if $0=y_{0}<y_{1}<\cdots<y_{n-1}<y_{n}=S_{\Gamma}$ is a partition of $\left[0, S_{\Gamma}\right]$, and because of the uniform continuity of $h, h^{-1}$, given $\varepsilon>0$ and a partition, there is a $\delta>0$ such that if the norm of the original partition is $<\delta$, then the norm of the induced partition is $<\varepsilon$.

Let $\{E(z): z \in C\}$ be a spectral family on $C$, and for $w=f(z)$, let $F(w)=E(z)$.

(5.51) LEMMA. $\{F(w): w \in \Gamma\}$ is a spectral family. 
Proof. Conditions (i)-(v) of Definition (2.2) are satisfied, using the uniform continuity of $h, h^{-1}$.

Let $T$ be the well-bounded operator defined by $T=\int_{C}^{\oplus} z d E(z)$.

(5.52) THEOREM. If $f \in \mathrm{AC}(C)$, then $f(T)=\int_{C}^{\oplus} f(z) d E(z)$ is well-bounded.

Proof. By the functional calculus [21, p. 636], $f(T)$ is defined. Since $\{F(w)\}$ is a spectral family, $V=\int_{\Gamma}^{\oplus} w d F(w)$ is a well-bounded operator. It suffices to show that $V=f(T)$. If $\left\{y_{i}\right\}$ is a partition of $\left[0, S_{\Gamma}\right]$ with norm $\left|\left\{Y_{i}\right\}\right|$, and if $\left\{x_{i}\right\}$ is the induced partition of $\left[0, S_{C}\right]$, then by the definition of $\int^{\oplus}$ immediately preceding Proposition (2.3),

$$
\begin{aligned}
V & =\gamma F(\gamma)+\lim _{\left|\left\{y_{i}\right\}\right| \rightarrow 0} \sum_{1}^{n} \sigma\left(y_{i}\right)\left[F\left(\sigma\left(y_{i}\right)\right)-F\left(\sigma\left(y_{i-1}\right)\right)\right] \\
& =f(\alpha) E(\alpha)+\lim _{\left|\left\{x_{i}\right\}\right| \rightarrow 0} \sum_{1}^{n} f\left(\rho\left(x_{i}\right)\right)\left[E\left(\rho\left(x_{i}\right)\right)-E\left(\rho\left(x_{i-1}\right)\right)\right] \\
& =f(T),
\end{aligned}
$$

where we have used the fact that $\left|\left\{y_{i}\right\}\right| \rightarrow 0$ if and only if $\left|\left\{x_{i}\right\}\right| \rightarrow 0$.

(5.53) THEOREM. Let $n$ be even and let $L$ be an nth order Birkhoff regular differential operator with simple spectrum, such that $\alpha_{1} \neq \alpha_{2}$. Then for $t \geqslant 0$, each operator $U(t)$ in the semigroup generated by $-L$ is a well-bounded operator.

Proof. For given $t>0, f(\mu)=e^{t / \mu}$ is ultimately one-to-one on the admissible arc $C$ containing the $u_{k}$ 's. Since $f$ is also absolutely continuous, we see that $\int_{C}^{\oplus} f(\mu) d E(\mu)$ is well-bounded. Since

$$
U(t)=e^{\lambda t} \int_{c}^{\oplus} f(\mu) d E(\mu)
$$

it suffices to show that a scalar multiple of a well-bounded operator is well bounded. To this end, we note that the map $z \rightarrow z_{0} z$, for any fixed scalar $z_{0} \neq 0$, satisfies the conditions of Theorem (5.52), so if $T$ is well-bounded, so is $z_{0} T$.

In the case of semigroups generated by second order differential operators, it is possible to see further structure: each such semigroup is a semigroup of polar operators composed with a uniformly continuous semigroup.

Let

$$
P_{k} f=\left(f, \varphi_{k}\right) \varphi_{k}, \quad \varphi_{k}(x)=e^{i k x} .
$$

Since the spaces $L^{p}(-\pi, \pi)$ admit conjugation, the operators

$$
Q_{1}=\sum_{1}^{\infty} P_{k}, \quad Q_{2}=\sum_{-\infty}^{-1} P_{k}
$$

are everywhere defined and bounded. The translation operator

$$
(T(t) f)(x)=f(x+t)=\sum_{-\infty}^{\infty} e^{i k t} P_{k} f, \quad-\infty<t<\infty,
$$


can be expressed as $T(t)=e^{i A(t)}$ where $A(t)$ is a well-bounded operator on $L^{p}$ $(1<p<\infty)$, the spectrum of $A(t)$ is in [0,2 ], $2 \pi$ is not an eigenvalue of $A(t)$, and $A(t)$ is a multiplier transform

$$
A(t)=\sum_{-\infty}^{\infty} a_{k}(t) P_{k}, \quad 0 \leqslant a_{k}(t)<2 \pi .
$$

Furthermore, there is a constant $K_{p}>0,1<p<\infty$, such that if $E_{t}(\lambda)$ denotes the spectral family of $A(t)$, then $\left\|E_{t}(\lambda)\right\| \leqslant K_{p}, t, \lambda \in \mathbf{R}$. See the proposition in Example (4.47).

(5.54) Lemma. Let $b, d$ be fixed real constants, and let

$$
D_{0}(t)=Q_{1} A(b t)+Q_{2} A(d t) .
$$

Then $D_{0}(t)$ is well-bounded for each $t$. If $E_{t}^{0}(\lambda)$ denotes the spectral family for $D_{0}(t)$, then there is a constant $K_{p}>0,1<p<\infty$, such that

$$
\left\|E_{t}^{0}(\lambda)\right\| \leqslant K_{p}, \quad t, \lambda \in \mathbf{R} .
$$

Proof. Since $A(t)$ is well-bounded, for every polynomial $p$ on $[0,2 \pi],\|p(A(t))\|$ $\leqslant K_{t}\|p\|, K_{t}>0$. Since the $P_{k}$ 's are mutually orthogonal, we have

$$
p\left(D_{0}(t)\right)=p\left(Q_{1} A(b t)\right)+p\left(Q_{2} A(d t)\right)=Q_{1} p(A(b t))+Q_{2} p(A(d t))
$$

and

$$
\left\|p\left(D_{0}(t)\right)\right\| \leqslant\left(\left\|Q_{1}\right\| K_{b t}+\left\|Q_{2}\right\| K_{d t}\right)\|p\| .
$$

The uniform boundedness of $\left\|E_{t}^{0}(\lambda)\right\|$ follows from the similar property for $\left\|E_{t}(\lambda)\right\|$, and the relation $E_{t}^{0}(\lambda)=Q_{1} E_{b t}(\lambda)+Q_{2} E_{d t}(\lambda)$.

Let $L$ be a second order differential operator with eigenvalues $\lambda_{k}$, eigenfunctions $u_{k}$, and adjoint eigenfunctions $v_{k}$. Then the semigroup generated by $-L$ is

$$
U(t) f=\sum_{-\infty}^{\infty} e^{-\lambda_{k} t}\left(f, v_{k}\right) u_{k} .
$$

Since the $u_{k}$ 's are equivalent to the $\varphi_{k}$ 's: $u_{k}=B \varphi_{k}$, we have $U(t)=B U_{0}(t) B^{-1}$, where $U_{0}(t)=\sum_{-\infty}^{\infty} e^{-\lambda_{k} t} P_{k}$. Using (5.20), we see that there are real constants, $a, b, c, d$, and a complex sequence $\left\{\delta_{k}\right\}$ in $l^{2}$, such that

$$
\begin{cases}\lambda_{k}=k^{2}+a k-i b k-\delta_{k}, & k \rightarrow+\infty, \\ \lambda_{k}=k^{2}+c k-i d k-\delta_{k}, & k \rightarrow-\infty .\end{cases}
$$

Since $\sum \delta_{k} P_{k}$ is a bounded operator in $L^{p}(1<p<\infty)$, we see that $S_{0}(t)=\sum e^{\delta_{k} t} P_{k}$ is a uniformly continuous semigroup. From (5.55), we have $U_{0}(t)=V_{0}(t) S_{0}(t)$, where $V_{0}(t)=R_{0}(t) X_{0}(t)$, and

$$
\begin{aligned}
& R_{0}(t) F=P_{0}+\sum_{1}^{\infty} e^{-\left(k^{2}+a k\right) t} P_{k}+\sum_{-\infty}^{-1} e^{-\left(k^{2}+c k\right) t} P_{k}, \\
& X_{0}(t)=\sum_{1}^{\infty} e^{i k b t} P_{k}+\sum_{-\infty}^{-1} e^{i k d t} P_{k} .
\end{aligned}
$$


Since the exponentials in $R_{0}(T)$ are ultimately monotone, $R_{0}(t)$ is a well-bounded operator.

(5.57) LeMMA. $X_{0}(t)=e^{i D_{0}(t)}$.

Proof. From (5.56), we have $X_{0}(t)=Q_{1} T(b t)+Q_{2} T(d t)$. Now

$$
D_{0}(t)=\sum_{1}^{\infty} a_{k}(b t) P_{k}+\sum_{-\infty}^{-1} a_{k}(d t) P_{k}
$$

and

$$
\begin{aligned}
e^{i D_{0}(t)} & =\sum_{1}^{\infty} e^{i a_{k}(b t)} P_{k}+\sum_{-\infty}^{-1} e^{i a_{k}(d t)} P_{k} \\
& =Q_{1} T(b t)+Q_{2} T(d t) .
\end{aligned}
$$

(5.59) THEOREM. Let $L$ be a second order Birkhoff regular differential operator with simple spectrum, such that $\alpha_{1} \neq \alpha_{2}$. Let $U(t)$ be the semigroup generated by $-L$ on $L^{p}(-\pi, \pi), 1<p<\infty$. Then

$$
U(t)=R(t) e^{i D(t)} S(t),
$$

where $\left\{R(t) e^{i D(t)}\right\}$ is a semigroup of polar operators satisfying the hypothesis of Theorem (4.5), and $S(t)$ is a semigroup continuous in the uniform operator topology.

Proof. Let

$$
R(t)=B R_{0}(t) B^{-1}, \quad D(t)=B D_{0}(t) B^{-1}, \quad S(t)=B S_{0}(t) B^{-1} .
$$

Clearly $R(t), D(t)$ are well bounded, and since they are multiplier transforms, they commute. It is also clear that the spectrum of $R(t)$ is nonnegative. If $F_{t}(\lambda)$ denotes the spectral family of $R(t)$, then $F_{t}(0)=0$ since $R(t)$ is one-to-one, so $F(0) e^{i D(t)}=$ $F(0)$. Since the well-boundedness of $D_{0}(t)$ is implemented by the interval [0,2 $]$ ], this interval contains the spectrum of $D(t)$. To see that $2 \pi$ is not in the point spectrum of $D(t)$, we use the representation (5.58) and the fact that $a_{k}(t)<2 \pi$ for all $k$ and all $t$. It is evident that $\left\{U(t) S^{-1}(t)\right\}$ is a strongly continuous semigroup. Thus $\left\{R(t) e^{i D(t)}\right\}$ is a strongly continuous semigroup and each $R(t) e^{i D(t)}$ is a polar operator written in its canonical decomposition. The final hypothesis of Theorem (4.5) is the uniform boundedness of the spectral families of $D(t), t \in \mathbf{R}$. This follows from Lemma (5.54).

(5.60) THEOREM. Let L be as in the previous theorem. Then there exists a bounded operator $M$ on $L^{p}(-\pi, \pi), 1<p<\infty$, such that $-(L+M)$ generates the polar semigroup $R(t) e^{i D(t)}$.

Proof. Using (5.55) in the spectral decomposition of $L$,

$$
L=B \sum_{-\infty}^{\infty} \lambda_{k} P_{k} B^{-1},
$$

we see that $M=B \sum \delta_{k} P_{k} B^{-1}$. 


\section{REFERENCES}

1. J. Y. Barry, On the convergence of ordered sets of projections, Proc. Amer. Math. Soc. 5 (1954), 313-314.

2. H. E. Benzinger, The $L^{p}$ behavior of eigenfunction expansions, Trans. Amer. Math. Soc. 174 (1972), $333-344$.

3. Pointwise and norm convergence of a class of biorthogonal expansions, Trans. Amer. Math. Soc. 231 (1977), 259-271.

4. A A canonical form for a class of ordinary differential operators, Proc. Amer. Math. Soc. 63 (1977), 281-286.

5. __ Eigenvalues of regular differential operators, Proc. Roy. Soc. Edinburgh Sect. A 79 (1977), 299-305.

6. G. D. Birkhoff, Boundary value and expansion problems of ordinary linear differential equations, Trans. Amer. Math. Soc. 9 (1908), 373-395.

7. P. L. Butzer and H. Berens, Semi-groups of operators and approximation, Die Grundlehren der math. Wissenschaften, Vol. 145, Springer-Verlag, New York, 1967.

8. I. Colojoară and C. Foiaş, Theory of generalized spectral operators, Gordon \& Breach, New York, 1968.

9. H. R. Dowson, Spectral theory of linear operators, London Math. Soc. Mono., No. 12, Academic Press, New York, 1978.

10. N. Dunford and J. T. Schwartz, Linear operators. I, General theory, Pure and Applied Math., Vol. 7 , Interscience, New York, 1958.

11. R. E. Edwards and G. I. Gaudry, Littlewood-Paley and multiplier theory, Ergebnisse der Math. und ihrer Grenzgebiete, Vol. 90, Springer-Verlag, New York, 1977.

12. T. A. Gillespie, $A$ spectral theorem for $L^{p}$ translations, J. London Math. Soc. (2) 11 (1975), 499-508.

13. T. A. Gillespie and T. T. West, Weakly compact groups of operators, Proc. Amer. Math. Soc. 49 (1975), 78-82.

14. E. Hille and R. S. Phillips, Functional analysis and semi-groups, Amer. Math. Soc. Colloq. Publ., vol. 31, Amer. Math. Soc. Providence, R.I., 1957.

15. E. Hille and J. D. Tamarkin, On the theory of Fourier transforms, Bull. Amer. Math. Soc. 39 (1933), $768-774$.

16. K. Hoffman, Banach spaces of analytic functions, Prentice-Hall, Englewood Cliffs, N.J., 1962.

17. Y. Katznelson, An introduction to harmonic analysis, Wiley, New York, 1968.

18. M. A. Neumark, Lineare Differentialoperatoren, Akademie-Verlag, Berlin, 1960.

19. D. J. Ralph, Semigroups of well-bounded operators and multipliers, Thesis, Univ. of Edinburgh, 1977.

20. F. Riesz and B. Sz. Nagy, Functional analysis, Ungar, New York, 1955.

21. J. R. Ringrose, On well-bounded operators. II, Proc. London Math. Soc. (3) 13 (1963), 613-638.

22. S. Salaff, Regular boundary conditions for ordinary differential operators, Trans. Amer. Math. Soc. 134 (1968), 355-373.

23. D. R. Smart, Conditionally convergent spectral expansions, J. Austral. Math. Soc. 1 (1960), 319-333.

24. A. R. Sourour, Semigroups of scalar type operators on Banach spaces, Trans. Amer. Math. Soc. 200 (1974), 207-232.

25. M. H. Stone, A comparison of the series of Fourier and Birkhoff, Trans. Amer. Math. Soc. 28 (1926), 695-761.

26. E. C. Titchmarsh, The theory of functions, 2nd ed., Oxford Univ. Press, Oxford, 1939.

Department of Mathematics, University of IllinoIs, Urbana, Illinois 61801

Department of Mathematics, University of Edinburgh, Edinburgh, EH9 3JZ, Scotland 